\title{
GRPANL: a program for fitting complex peak groupings for gamma and $x$-ray energies and intensities
}

Raymond Gunnink Wayne D. Ruhter

January, 1980

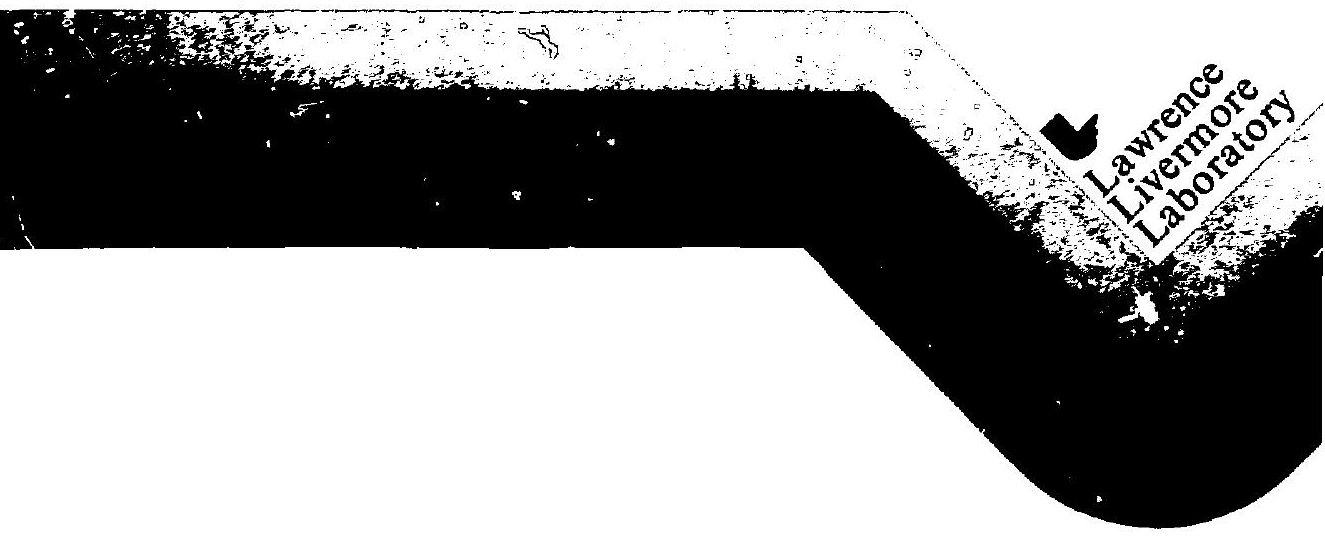


LCRL-52917

Distribution Category UC-10

\title{
GRPANL: a program for fitting complex peak groupings for gamma and $x$-ray energies and intensities
}

\author{
Raymond Guanink \\ Wayne D. Ruhter
}

Manuscript date: January, 1980

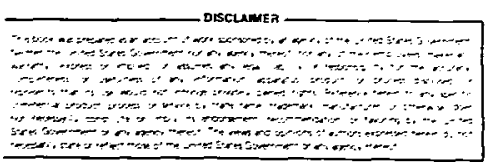

LAWRENCE LIVERMORE LABORATORY University of California $\bullet$ Livermore, California 94550 


\section{CORTENTS}

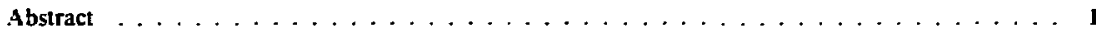

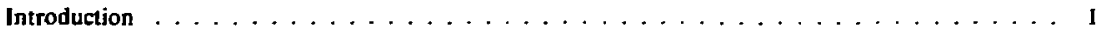

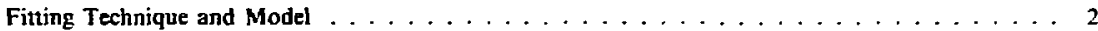

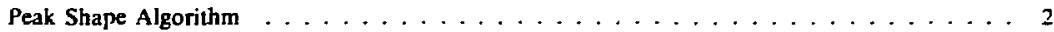

Delineation of Backgrcund . . . . . . . . . . . . . . . . . . . . 4

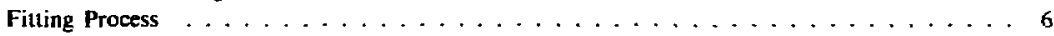

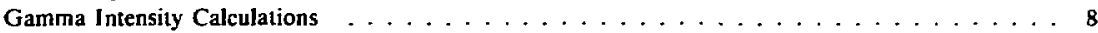

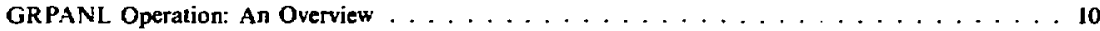

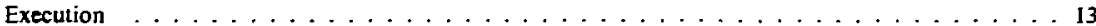

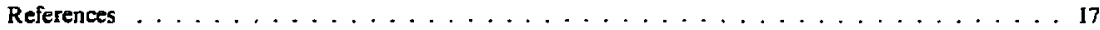

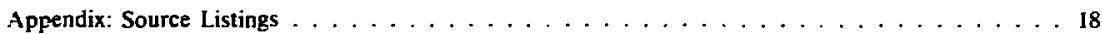




\title{
GRPANL: a program for fitting complex peak groupings for gamma and $x-$ ray energies and intensities
}

\begin{abstract}
APSTRACT
GRPANL is a general-purpose peak-fitting program that calculates gamma-ray and $x$-ray energies and intensities from a given spectral region. The program requires that the user supply input information such as the first and last channels of the region, the channels to be used as pre- and post-region background, the system gain and zero-intercept, and a list of approximate energy values at which peaks occur in the region. Because the peak position and peak-shape parameters enter nonlinearly into the peak-fitting algorithm, an iterative least-square procedure is used in the fitting process. The program iterates until either all convergence criteria are met or ten iterations have elapsed.

The code described here allows for twenty free parameters and a region as large as 240 data channels. This code runs on an LSI-11 computer with $32 \mathrm{~K}$ memory and disk-storage capability.
\end{abstract}

\section{INTRODUCTION}

The program cailed GRPANL (GRouP ANaLysis) first developed in 1974, has evolved as an interactive program for use on minicomputers to analyze peak groupings of gamma and $x$ rays in spectra taken with germanium detectors. Its purpose is to calculate peak positions, or energies, and peak intensities while allowing the operator control with respect to the initial parameters for analysis of the peak grouping. Recently, the program has been adapted by others to perform automatic, though prescribed, analyses of spectra. ${ }^{1.2}$

We describe here the algorithm used by GRPANL and provide a Fortran listing of the source code. Although the program was originally written for use on a PDP-8 computer, the version given in the Appendix of this report operates on a PDP-11 under Digital Equipment Corporation's RT-11-V03 operating system. GRPANL, with its subpeograms ULOAD2, ULFIT2, and ULOUT¿. is capable of analyzing a peak grouping spanning a region of up to 240 channels and allows up to 20 parameters to be freed, i.e. degrees of freedom allowed with respect to any combination of peak heights, positions, and peak shape parameters. The operator may tie any peak position or intensity to that of another designated peak within a region. $X$ rays, whose shapes differ from gamma rays, can also be fitted. The peak energies are determined either by specifying the exact gain and zerointercept, or by allowing calculation of the eneigy scaling, using specified internal reference peaks. For exact energy determinations, the program accounts for system nonlinearity. Finally, GRPANL can calculate gamma emission rates from peak areas by means of efficiency algorithms, which are based on the same model used by the generalized computer program GAMANAL. ${ }^{3.4}$ which is a comprehensive program that not only reduces but interprets data. 


\section{FITTING TECHNIQUE AND MODEL}

Accurate determination of peak positions and areas is one of the most important aspects of analyzing complex $x$ - and gamma-ray spectra obtained with solid-state detectors. These measurements usually require a fitting procedure, particularly when two or more peaks overlap severely. Several methods of fitting have been developed. ${ }^{47}$ Each fitting technique is based on a supporting model or methodology. The methodology frequently grows out of such factors as experience. intended use of the analysis program, size and speed of the computer used, and accuracy that is required. In this section, we will discuss the methodology used in the GRPANL program.

\section{PEAK SHAPE ALGORITHM}

The most easily detected and intense portion of a gamma-ray peak may be described by a Gaussian function, as illustrated in Fig. 1. However, deviations from the Gaussian distribution known as tailing occur on the low-energy and occasionally on the high-energy side of a peak. The low-side tailing is a result of incomplete charge collection in the detector, though low-angle scattering in the sample source or in surrounding materials can also contribute to low-side tailing. Tailing on the highenergy side arises from signal distortion by the pulse electronics modules. This latter effect should be eliminated or reduced as much as possible.

The tailing portion of a peak can generally be described by means of one or more terms of exponential form. Some computer programs divide each peak into regions and apply a separate fitting function to each region. ${ }^{5}$ The boundary conditions are chosen so that the calculated response and, in most cases its first derivative, form a smooth curve. The analytical expression for the shape function ssed by GRPANL is adapted from the work of Sanders and Holmes ${ }^{8}$ and treats the entire peak region with a single expression:

$$
y_{i}=y_{0}\left[\operatorname{expoc}\left(x_{i}-x_{0}\right)^{2}+T(x)\right]
$$

where

$$
y_{i}=\text { net counts in channel } x_{j} \text { for a single }
$$
peak

$$
\begin{aligned}
y_{0}= & \text { peak height } \\
\alpha= & -1 / 2 \sigma^{2}=-2.7726 /(\text { FWHM })^{2}, \text { where } \\
& \text { FWHM is the variable containing the } \\
& \text { peak-width } \\
x_{0}= & \text { peak position } \\
T(x)= & \text { tailing function. }
\end{aligned}
$$

The tailing function is given by

$$
\begin{array}{r}
T(x)=\left[A \cdot \exp B\left(x_{i}-x_{0}\right)+C \cdot \exp D\left(x_{i}-x_{0}\right)\right] \\
\times\left[1-\exp 0.4 \alpha\left(x_{i}-x_{0}\right)^{2}\right] \delta
\end{array}
$$

where

$$
\begin{aligned}
& A \text { and } C=\text { the short-and lon,-term tailing am- } \\
& \text { plitudes, } \delta \text { illustrated in Fig. } 1 . \\
& \text { B and } D=\text { the tailing slopes } \\
& \text { and } \delta=1 \text { for } x_{i}<x_{0} ; \delta=0 \text { for } x_{i}>x_{0} .
\end{aligned}
$$

The expression in the right-hand bracket of the tailing function smoothly reduces the tailing intensity to zero as the peak centroid is approached. The long-term tailing component is disregarded (i.e., C $=0$ ) for many applications. However, inclusion of this component is desirable in order to obtain a more accurate fit of a large-peak multiplet having good counting statistics.

The peak-shape parameters in the above expression are $y_{0}, x_{0}, \alpha, A, B$, and occasionally $C$ and D. Peak positions and heights are generally the measurements of interest, and therefore $x_{0}$ and $y_{0}$ are the principal parameters in the fitting process. The other parameters may be treated as fixed or as free parameters, depending on whether they have been predetermined by calibration. We have found that the values of $\alpha, A$, and B can be expressed by simple equations involving the gamma-ray energy.

For example, the peak width $\sigma_{T}$ can be expressed as a function of the gamma-ray energy $E$ hy

$$
\sigma_{T}^{2}=\sigma_{n}^{2}+\sigma_{s}^{2}=k_{1}+k_{2} E
$$

where $\sigma_{n}$ is related to the system "noise," and $\sigma_{s}$ is a term related to the statistical nature of the charge collection process. Hence, the measured width of 


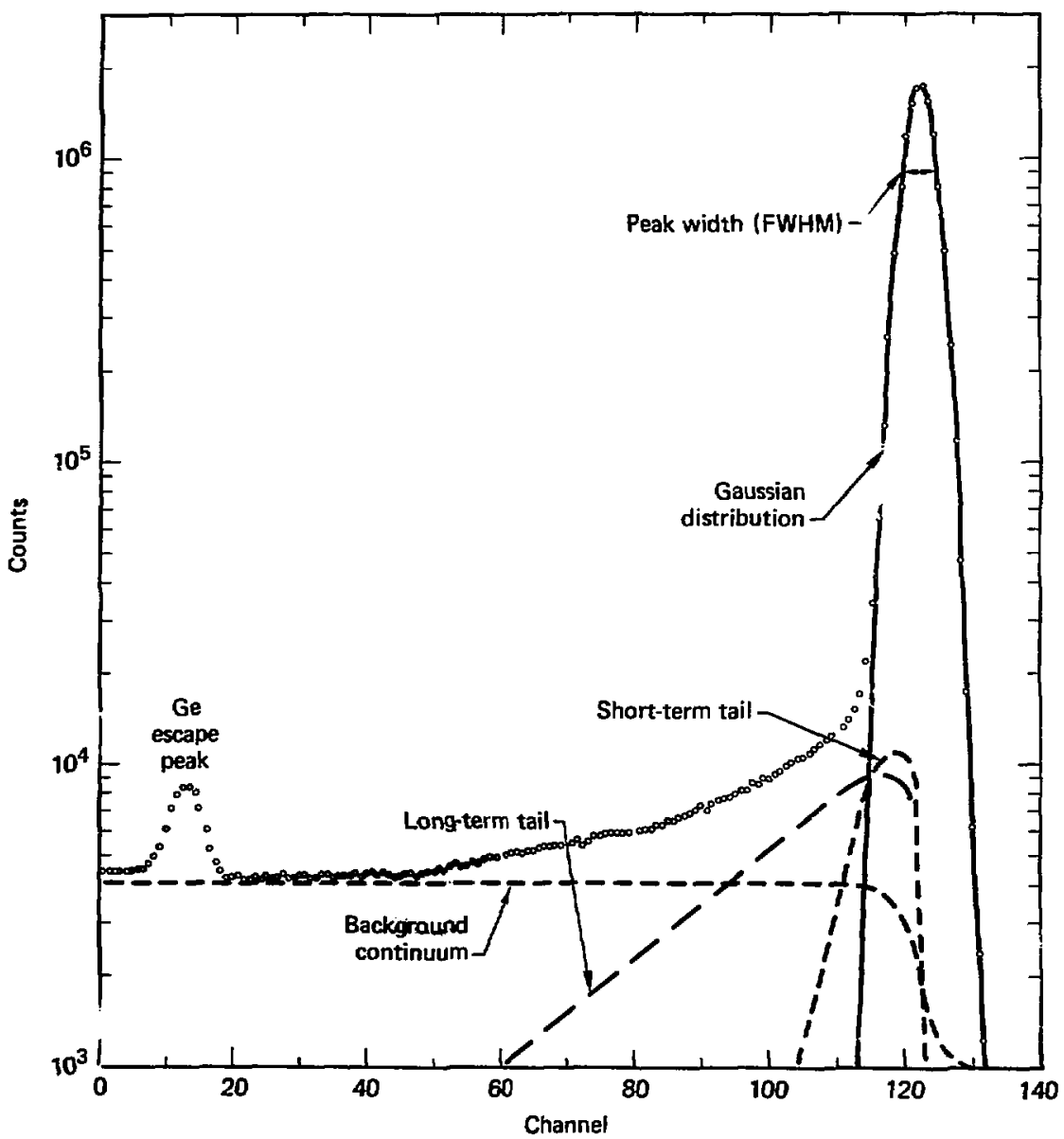

FIG. 1. The primcipal conponent of the peak distribution is described by a Gaussian function. The low-side tailing can be described by two adjitional fumctions. 
two isolated peaks, preferably a low- and a highenergy peak. can be used to determine the values for coefficients $k_{1}$ and $k_{2}$. The above expression can subsequently be used to calculate the width parameter for a peak of any other energy.

By experience we have found that the tailing slope $B$ can generally be treated as a constant for a given detector system. For an accurate determination of $\mathrm{B}$, a high-energy peak where the tailing is most severe should be used. The peak should be caused by a single gamma ray and have good counting statistics. Once B is determined and held constant, the tailing amplitude $A$ can then be expressed by:

$$
\ln A=k_{3}+k_{4} E
$$

The same two peaks that were used to determine the values for $k_{1}$ and $k_{2}$ determine $k_{3}$ and $k_{4}$

There are now five basic peak-shape constants: $k_{1}, k_{2}, k_{3}, k_{4}$, and $B$, plus the two long-term tailing constants. $C$ and $D$, required only in special cases. GRPANL allows entry of the name of a disk file of peak-shape constants. The operator may still control whether the parameters calculated using these shape constants will be fixed in the fitting process or whether they will ouly be used as a starting estimate toward improving its value. If a disk file containing shape constants is not constructed the operator must provide initial estimates of the parameters, that is, estimates for the peak width (FWHM), the tailing amplitude $A$ and the tailing slope $B$.

The peak shapes of $x$ rays differ from those of gamma rays of the same energies because atomic transitions have much shorter lifetimes than nuclear transitions. This difference becomes more pronounced with increasing energy. The intrinsic radiative width of heavy-element $x$ rays at $100 \mathrm{keV}$ is about $100 \mathrm{eV}$, whereas gamma-ray widths are typically a small fraction of an electron volt. Furthermore, the intrinsic distribution is Lorentzjan in shape. When the broadened $x$-ray distribution is convoluted with the nearly Gaussian instrumental response, the result is a more complex peak shape than that produced by gamma rays.

The modification to the peak shape algorithm necessary to fit an x-ray distribution, illustrated in Fig. 2, is discussed in an earlier report. ${ }^{9}$ GRPANL uses this modified algorithm.

\section{DELINEATION OF BACKGROUND}

The peaks of any $x$-or gamma-ray spectrum lie on top of a background distribution resulting from the Compton scattering process. In GRPANL, this distribution must be removed before the peaks can be fitted. This is done by specifying regions on either side of the peak grouping that establish corresponding background levels. The operator may also specify the slopes of the background continuum preceding and following the peak grouping if a peak grouping rests on a sloping background.

An explicit function ${ }^{4}$ calculates the interpolated background values under the peak grouping. This function has the following form:

$$
B_{i}=b_{n}+\left(b_{m}-b_{n}\right)\left(\sum_{j=n}^{j=i} y_{j}\right)
$$

where

$B_{i}=$ computed background at channel $i$,

$y_{i} \quad=$ spectrum count of channel $i$,

$b_{n}$ = average background level of the lowenergy side of the peak,

$b_{n}=$ average background level of the highenergy side of the peak.

This function produces smoothed steps in the background that occur at positions of greatest intensity. This procedure works equally well for single peaks and for complex peak groupings, an example of which is shown in Fig. 3. An examination of Eq. (5) shows that it is essentially equivalent to the error-function form ${ }^{6}$ for describing the background contin.? Im. However, the function in Eq. (5) is used to remove the background before the fitting process commences, whereas the error-function method includes it in the fitting process. The latter method therefore requires more fitting parameters and greater calculational time.

If a background continuum slope has been specified, the steps in the background are superimposed on the specified slope, and the size of the steps is increased or decreased depending on the sign and steepness of the slope. If the two input 


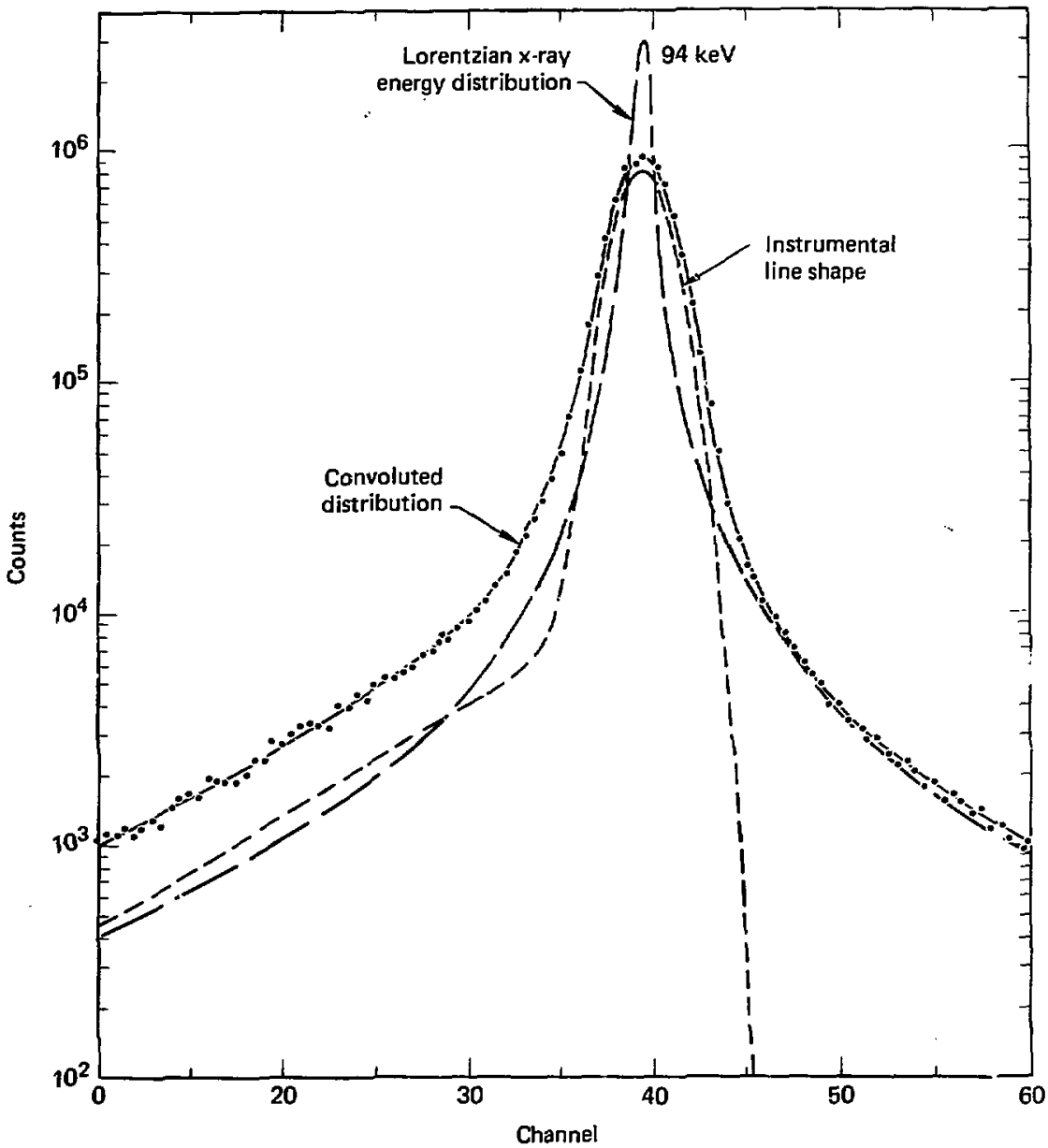

FIG. 2. The intrinsic $x$-ray energy distribution is Lorentzian in shape. This distribution convolutes with the instramental line shape resulting in - modified peak shape. 


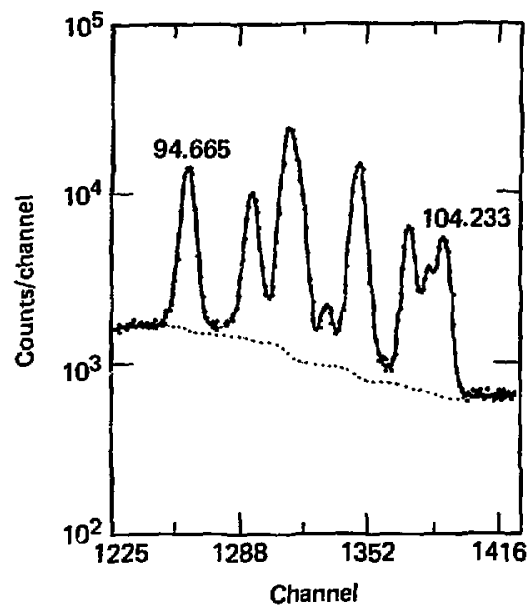

FIG. 3. The background coatimum for a complex moltiplet as showa here may be accurately calculated by the explicit function given in Eq. (5).

stopes are different, the background continuum makes a gradual change from the one to the other.

The selection of the group boundary position is usually a matter of judgement, and it is frequently difficuit to determine or define where a peak should start or end. This is particularly true if one is to include long-term tailing and/or $x$ rays in the fitting process, since these distributions extend to considerable distances from the peak, as previously illustrated in Figs. 1 and 2.

Regardless of where tbe boundaries are chosen, the regions selected for measuring the average background levels usually contain small contributions from the peaks. GRPANL handles this problem by adjusting the measured levels according to the current value of the contribution of the peaks to the two background regions. The adjustment is refined after each iteration of the least-squares fitting process.

Although long-term tailing may be included in the fitting process, GRPANL does not include the area beneath the long-term tailing as part of the reported peak area. However, the area under the short-term tail is included. From experience, we recommend that a short-term tailing slope of $B=1$ be used to distinguish the two forms of tailing. The short-term tailing slope should usually have a value between 1.0 and 5.0. The tailing amplitude varies accoriting to the slope value with high amplitude occurring with higher slope values. It also varies according to the magnitude of peak tailing with low energy peaks of good detectors exhibiting rather low values (0.01-0.1) and higher energy peaks exhibiting tailing amplitude values greater than 1.0.

\section{FITTING PROCESS}

Following the removal of the background continuum, the net counts in each channel of a peak multiplet are considered as a set of data values $Y_{i}$ that have been measured with respect to an independent variable $x_{i}$ and to which several overlapping single peaks may be contributing according to the expression

$$
Y_{i}=\sum_{j=1}^{n} y_{j i}
$$

where $n$ is the number of peaks in the grouping.

We now must find a function which, when evaluated point by point, most closely approximates the original data values $\left(Y_{j}\right)$. This evaluation is done by minimizing chi square $\left(X^{2}\right)$. However, because thi: equations describing the peak shape are not of a linear form, an iterative procedure is used to arrive at a solution to the fit. The method used in GRPANL is a non-linear least-squares technique based on linearizing the equations by performing a first-order, Taylor's series expansion about the trial values of the free parameters. This procedure is also known as the Gauss-Seidel or Newton-Raphson method. From Eqs. (1) and (2), we see that $y_{i}$ is related to several peak parameters which we here designate simply as $\mathrm{pk}_{\mathrm{k}}$.

That is,

$$
y_{i j}=f\left(p_{1}, p_{2} \ldots p_{k}\right)
$$

By retaining only the first term of the Taylor's expansion of this equation, we derive

$$
y_{i j}-f\left(p_{1}{ }^{\circ}, p_{2}{ }^{\circ}, \ldots, p_{k}{ }^{\circ}\right)=\sum_{k=1}^{n}\left(\frac{\partial f}{\partial p_{k}}\right) \delta p_{k}
$$


where

$$
\begin{aligned}
y_{i j}= & \text { the contribution of the } j \text {-th peak to chan- } \\
& \text { nel } i \\
p_{\mathbf{k}}= & \text { current best estimate in the } k-t h \text { peak } \\
& \text { parameter } \\
\Delta \mathrm{p}_{\mathbf{k}}= & \text { the change required in the current best } \\
& \text { estimate of the } k \text {-th parameter to obtain } \\
& \text { a better fit to the data. }
\end{aligned}
$$

The current "free" parameter values are updated following each iteration, and the process is contirised until the current change in each parameter is less than the prescribed amount.

The rate of convergence depends upon a number of factors. Among these are the complexity of the grouping, the number of free parameters. the convergence criteria, and the accuracy of the initial parameter estimates. In addition to slow convergence, poor parameter estimates may also lead to an ill-conditioned matrix or a fitting sequence that does not converge. GRPANL precludes or rectifies thrse conditions by including boundary constraints. damping or limiting the estimated changes in the free parameters and limiting the allowable number of iterations to 10 .

Convergence is determined by comparing the magnitude of the last changes made in each of the parameters being determined with internally specified convergence criteria. Each convergence criterion value is calculated using an equation that contains a fixed and a variable component, the latter being related to the error associated with the parameter being determined. The use of the error component allcws early convergence because the specification arising only from the fixed component would be needlessly tight when applied to statistically poor data.

Once convergence has been attained, final calculations are made for peak energies, intensities, and the respective errors. The latter are based on counting statisiics and on the goodness-of-fit (the variable QFIT)* and are normally calculated using the formula

$$
\text { ERROR }(j)=(D M(j) * Q F I T)^{1 / 2}
$$

where

$$
\operatorname{DM}(j)=\underset{\text { diagonal error matrix element of }}{\text { parameter } j}
$$

and

$$
\begin{aligned}
& \text { QFIT }=\left(\sum_{i=1}^{n} R_{i}^{2}\right) / D O F \\
& R_{i}=\text { the weighted residuals of the fit }
\end{aligned}
$$

and

$$
\text { DOF }=\text { degrees of freedom. }
$$

With this method, a poor fit in one region affects all the calculated errors, whereas intuitively we know that this is not necessarily true. We have therefore modified Eq. (10) so that a "localized" goodness-offit is calculated and applied to each peak in the multiplet. In the new expression, each $\mathbf{R}_{\mathbf{k}}{ }^{2}$ value is appropriately weighted according to its proximity to the peak in question.

Thus

$$
Q F I T(j)=\frac{\sum_{i=1}^{n} R_{i}^{2}\left[\exp \left(\alpha \cdot \Delta x_{i}^{2}\right)\right]^{1 / 2}}{\sum_{i=1}^{n}\left[\exp \left(\alpha \cdot \Delta x_{i}^{2}\right)\right]^{1 / 2}}
$$

where

$$
\begin{aligned}
& 1 \quad=\text { peak width parameter } \\
& \Delta \mathrm{x}_{\mathrm{i}}=\text { distance from peak position. }
\end{aligned}
$$




\section{GAMMA INTENSITY CALCULATIONS}

GRPANL also calculates gamma emission rates from the determined peak areas. The instructions are based on the same cfficiency model that is used in the GAMANAL program. ${ }^{3,10} \mathrm{~A}$ basic premise of the model is that the overall efficiency can be treated as the jroduct of two components: the intrinsic efficiency of the detector, defined in Eq. (12), and the source-detector geometry. The latter is calculated after r.etermining two hypothetical points in space, one at which the detector is said to reside and another at which all of the sample is said to be concentrated. To determine the efficiency for a particular source-cetector geometry, the rule of physics is invoked; that the detection efficiency is inversely proportional to the square of the sourse-todetector distance. The fundamental equation used by the model is

$$
\epsilon_{\mathrm{E}} \equiv \frac{\mathrm{C}_{\mathrm{E}}}{\gamma_{\mathrm{E}}} \cdot \mathrm{d}^{2}
$$

where

$$
\begin{aligned}
\mathrm{E}_{\mathrm{E}}= & \text { counting efficiency for gamma ray of } \\
& \text { energy } \mathrm{E}, \text { normalized to } 1 \mathrm{~cm} \\
C_{\mathrm{E}}= & \text { net counts per unit time in peak } \\
\gamma_{\mathrm{E}}= & \text { source enission rate of gamma energy } \mathrm{E} \\
\mathbf{d}= & \text { source-to-detector distance. }
\end{aligned}
$$

Once a calibration curve ( $\epsilon$ as a function of E) has been determined, the equation can be transposed to yield photon emission rates, $\gamma_{E}$, for sources of unknown intensity.

A typical configuration for a cyindrical detector and a point source is shown in Fig. 4. To use Eq. (12), the parameter $d$ must be assessed by using measured or calculated values shown in Fig. 4 for:

$$
\begin{aligned}
x= & \text { distance from source to detector window } \\
w= & \text { distance from window to active detector } \\
& \text { surface } \\
\mathbf{p}= & \text { effective penetration of gamma ray into } \\
& \text { detector } \\
r= & \text { effective interaction radius in the detector } \\
r_{0}= & \text { limiting value of } r \text { at low energy } \\
\mathbf{d}= & \text { distance from source to effective zone of } \\
& \text { interaction } \\
T= & \text { detector thickness } \\
R= & \text { detector radius. }
\end{aligned}
$$

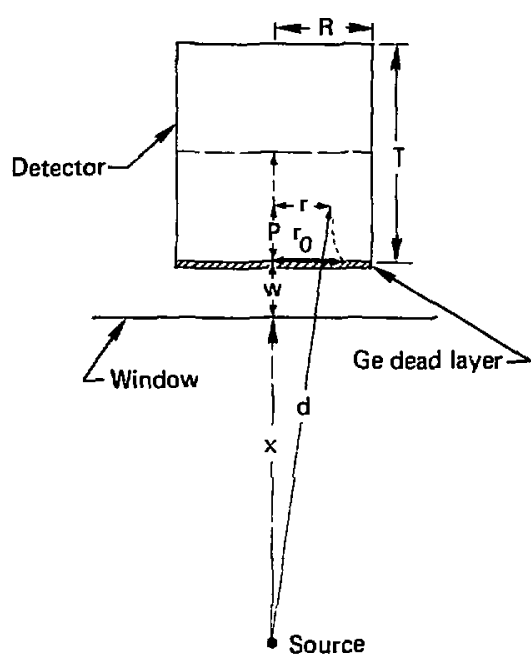

FIG. 4. A point source and a cylindrical detector used to model the effects of geometry on photopeak counting efficiency. Distances shown are defined in the section, Gamma Intensity Calculations.

From simple geometry we have

$$
d^{2}=(x+w+p)^{2}+r^{2} .
$$

Of these, $x$ is the only easily measured parameter. The others must be empirically determined, as discussed in Ref. 10. Our experience indicates that the geometry contribution to the counting efficiency for point sources can be calculated to within $\pm \mathrm{I}-2 \%$ of the correct values for all energies and all usable source-to-detector distances.

The intrinsic detection efficiencies as defined in Eq. (12) are determined by counting absolute standards at relatively large distances and normalizing these efficiencies to a $1-\mathrm{cm}$ distance. These values may be described by a fifth- or sixth-orde: polynomial equation of the form

$$
\ln \varepsilon_{i}=\sum_{j=1}^{n} a_{j}\left(\ln E_{i}\right)^{(j-1)}
$$


where

$t_{j}=$ efficiency at the $i$ th energy

$E_{i}=$ gamma energy

$a_{j}=$ coefficients of the polynomial.

We have found that a single function cannot adequately describe the full range of energies of interest, $0.05-4.0 \mathrm{MeV}$. Thus, we use two polynomial equations that overlap in the $100-200 \mathrm{keV}$ energy region. After the data are fitted by a least-squares analysis, the resulting curves are exatnined in the region of overlap, and a crossover energy is chosen that gives the same efficiency by either equation. In this way, the efficiency polynomial can be fitted to the calibration to within $\pm 1-2 \%$.
Many sources are not point sources. The model also contains formulac to account for the perturbations such as by source extension in two or three dimensions, gamma ray attenuation by the matrix of a volume sample, and oy absorbers between the source and the detector. These corrections are also discussed in Ref. 10.

For many prospective users of GRPANL, the inclusion and use of the efficiency model may perhaps be ancillary to the principal purpose of the program, which is to obtain peak positions (energies) and areas. We will therefore not further describe in this report details on the use of the model. 


\section{GRPANL OPERATION: AN OVERVIEW}

GRPANL is written in FORTRAN IV for use on PDP-, 1 systems operating with Digitai Equip17ent Corporation's RT-11 va3 or RSTS/E V06B. Because of its size, the program is broken up into GRPANL and three subprog:ams: LLOAD2. ULFIT2 and LIOUT2. GRPANL begins the execution, and control to the other programs is transferred directly by the subrouline CHAIN, found in the atrove-named system's subroutine library. The size of common biocks GNL and PASS prevents then from being iransferred automatically by the CHAIN subrouliue. The trancfer is accomplisined by each subprogram which writes the contents of the common blocks into the files GNL.DAT and PASS.DAT on the system derice before calling CHAIX. After transfer to the next suiprogram is completed, the subprogram reads back into cort the respective common blokk. The use: musi make available at least 24 bjoiks of cantiguous disk space on the system device befcre starting GRPANL for this operation to succeed.

The input data for the program is contained in tro disk files: the peak-shape paramster file and the spectral data file. The peak-shape parameter file contains the five basic peak-shape constants, $k_{1} \cdot k_{2}$, $k_{2} k_{f}$ and $B$. and the two long-term tailing constants, $C$ and $D$, which are necessary only in special cases. These constants may be calculated for a detector system by the progiam PKSHAP, listed in the Appendix. Input to PKSHAP requires the measured widths and tail amplitudes of two isolated peaks in a spectrum taken with the detector system. GRPA: $\mathrm{L}$ may be used to initially determine these param.eters. If a peak-shape paraneter file does not exist. GRPA NL requests the operator to provide initial estimates of the peak widtb (stored in variable FWHM). tailing amplitude (EXPI), and tailing slop: (EXP2). The new valies obtained by GRFANL are then used as input to PKSHAP, provi ied the value of QFIT, as defined in Eq. (10) is reasonatle.

The user enters the snape constant results printed out by PKSHAP into a peak-shape parameter fi!: a file created and edited by the program FIJ ES, which is also listed in the s: ppendix. FILES is a general-purpose program for preparing or editing unformatted sequential files. With FILES, the iser erters the five shape constants sequentially into the first frie locations of the peak-shape parameter file. If the user wishes to use lorig-term lailing in the fitting process. then the two long-:erm tailing constants should be entered into locations 7 and 8 of the file. If a correction for system nonlinearity is to be made, the appropriate coefficients are entered into locations 11 through 15. These coefficients are determined by least-squares fitting a power-series polynomial to data where the dependent variable is energy, asd the independent variable is the corresponding channel location. The user enters in location 1 1-15 the coefficients beyond first-order divided by the first-order coefficient. i.e., gain.

The spectral data file contains the pulse-height data which is stored in staricard PDP-11 floatingpoint notation, two words per channel count. The ULOUT2 program. which uses the peak areas to calculate ganna emission rates, requizes the livetime (seconds) for the count in the first location of the data file. The second location in this file stores the time of the count, in decimal days. This is for informational purposes only, and it is not necessary for the operation of the code. The spectral data for the region indicated 10 tte GRPANL code is read into core by the subroutine RDSK, which is also listed in the Appendix.

GRPANL reads in the inpu: parameters from the terminal and from the detector parameter disk fle, if any. From this information the subpiogram calculates the initial values for the peak-shape parameters and seis all the appropriate flags that indicate fixed or free parameters. Program control is transferied next to ULOAD2, which loads the spectral daia for the region being fit. The subprogram ULOAD2 determines the net channel data by removing the background calculated by the subroutine BKGRD. From the net data, ULOAD2 determines the initial values for the peak positions and beights. After ULOAD2, ULFIT2 performs an iterative fit to the data. After each iteration. the program prints on the terminal the current peak positic $n$ and heights. The current values for the peak shape parameters are also printed if a complete output was initially requested by the user. The program continues its iteration until the cusrent indicated change in each free parameter is less than a prescribed amount, of until 10 iterations have occurred. 
When the fitting process is completied, program control is transferred to ULOUT2. The function of the subprogram ULOUT2 is to calculate the peak energies, peak areas, errors on the piak areas, and the value of the reduced chi-square. The program prints this information along with the input information given by the user and the values otiermined for the peak-shape parameters with their associated errors. If the user chose the option of a complete printout. ULOUT2 lists, chanzel by channel, the net counts to which the fit was made, the residuals (difference between net count and salculated count), and the standard deviation, as illustrated in Fig. 5 , an abbreviated listing of the output.

The ULOUT2 subprogram ran also calculate gamma emission rates from the determined peak areas. In the ULOUT2 listing in the Appendix. the FORTRAN statements necessary for this calculaLion are shown as comment statensents. If ULOUT2 is used. a third disk file of detector-parameter data is required. This file is also created using the formatting program FILES. Sirteen entries are needed for the detector-parameter file:

(1-6) The high-energy portion, of the detector's inirinsic efficiency curve.

(7-11) The low-energy portion of the detector's intrinsic efficiency curve.

(12) The crossover energy for the above two polynomial equations describing the efficiency vs energy curve for the detector.

(13) The deteclor depth (cm).

(14) The detect ior radius (cm).

(15) Surface distance, window to detector (cm).

(16) Germanium dead layer on the front surface of the detectors $\left(\mathrm{g} / \mathrm{cm}^{2}\right)$.

These sixteen values are read into the array DCNST. 


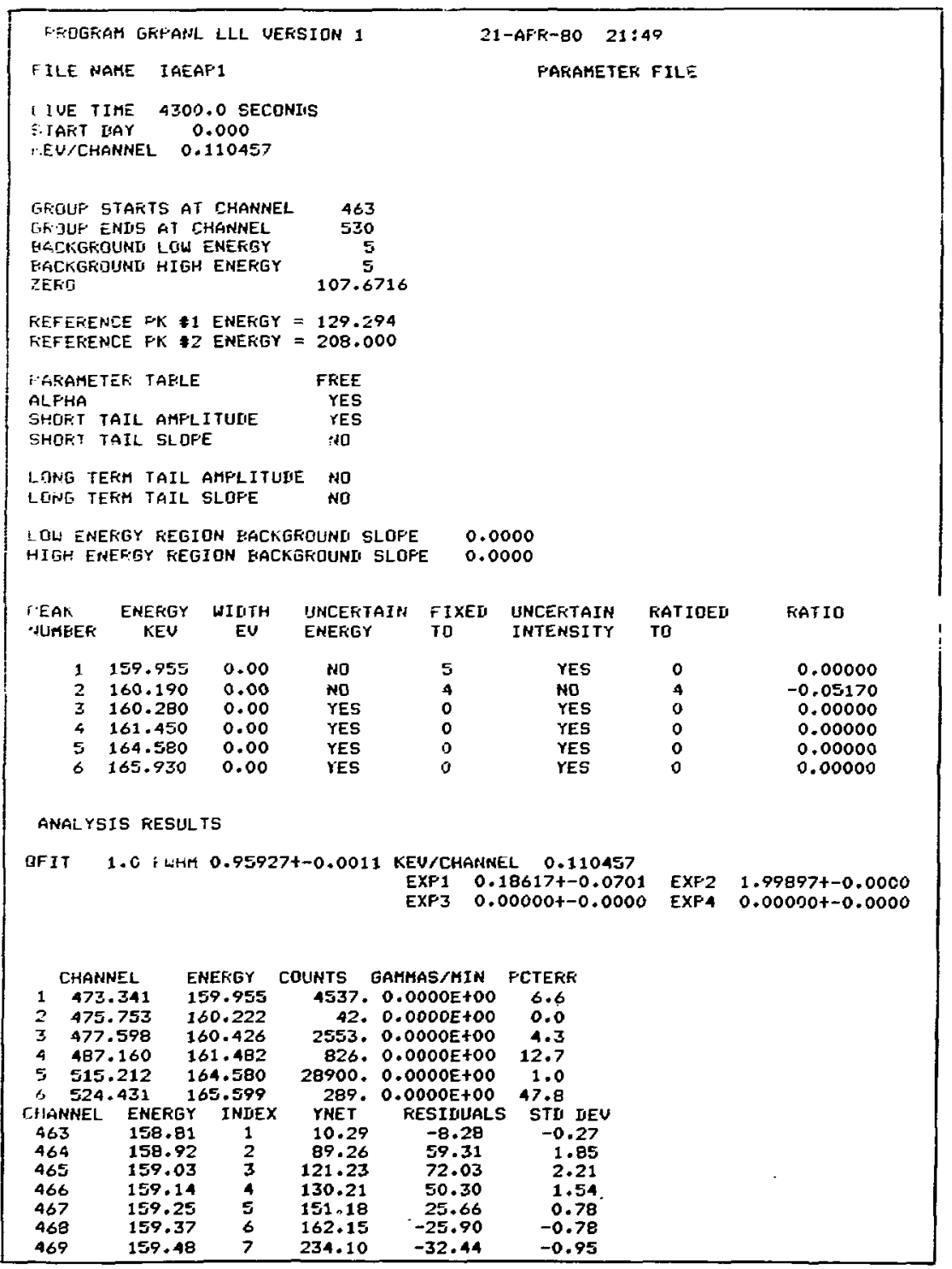

FIG.5. Fall ontpat from GRPANL res. 


\section{EXECUTION}

To begin execution of GRPANL, the user must have a file of spectral data on disk. An optional file of peak-shape parameters. and/or a detector parameter file may also be constructed prior to execution. $A$ carriage return must follow all input messages. File names have a maximum of six characters. which may be preceded by the device name and a colon if they do not reside on the default device. e.g. device:file.

User: R GRPANL

Routine: COMPLETE PRINTOUT?

User: $\quad Y \quad$ selects for inclusion in the output the position. net area, and error for each peak in the fit. and lists the net counts. calculated fil. residual (the difference between the net count and the calculated cotini $;$, and standard deviation for each channel in the region of interest. An abbreviated sample of the output is shown in Fig. 5.

N deletes channel by channel results from the report.

Routine: SPECTRUM ID IS

User: devicefile

specifies the file name of the spectral data file.

Routine: GROUP STARTS AT

User: $\quad$ nnnn $\quad$ specifies the first channel in the region of interest. for use by subroutine RDSK in filling array $Y$ from spectral data.

Routine: GROUP ENDS AT

User: nnnn speciffes the last channel in the region of interest. The number of channels between the starting and ending points may not exceed 240.

Routine: OF BKG. CHANNELS IN FRONT OF GROUP

User: nnnn specifies the number of channels preceding the starting channel to be used to calculate the background level on the low-energy side of the region.

Routine: $\%$ OF BKG. CHANNELS IN BACK OF GROUP

User: nnnn specifies the aumber of channels following the ending channel to be used in calculating the background level on the high-energy side of the region.

Routine: GAIN (NEG. VALUE FOR ABSOLUTE)

IF $=0$, PEAK AREA SIMPLY INTEGRATED

User: $\quad x \times \cdot x \times x \times x x$

(positive value) specifies the "approximate" gain used in locating reference peaks to determine the gain and zero for the spectrum. The energies of two well-defined peaks in 
the spectrum are then requested. The two peaks become calibration points for a twopoint determination of the gain and zero. If zero is entered for peak No. 1 (PK \#1). GRPANL assumes zero energy at channel zero.

ermines only the total net counts in the region. A zero entry signifies that a fit to the aks in the designated region not desired.

$-x x \cdot x \times x \times x x$

(negative value) specifies "absolute gain," i.e., no internal peaks are used for energy reference. and the final energy values are based on this gain.

\section{Routine: ENERGY OF ZERO CHANNEL}

User: $\quad x x x \cdot x \times x x$

specifies the energy of channel zero, information useful for those spectra which have a large zero offset.

Rouline: LIST PK ENERGIES IN THIS GROUP (NEG. VALUE FOR X-RAYS) TERMINATE WITH A ZERO

User: $\quad x \times x \times x \cdot x \times x \times$

specifies tbe energy for a peak in the region. Additional peak energies may be entered. each followed by a carriage return. Terminate the list of energies with a zero entry.

$-x \times x \times x, x \times x x$

(negative value) signifies that the peak is an $x$ ray. For negative values, the intrinsic width of the $x$-ray line of the form xxxx. $x$ is then entered after the prompt.

INTRINSIC WIDTH (EV)

IF ENTRY $=0$, AN INTERNALLY ESTIMATED VALUE USED

The values to be entered are listed in Ref. 11 . If zero is entered, an internal algorithm calculates an approximate width.

0 terminates the entry of peak energies.

Routine: PEAKS ENTERED FOR THIS REGION

(displays the number of peaks entered)

TYPE SEQUENCE \# OF PEAKS WHOSE ENERGY ARE UNCERTAIN

TYPE 99 FOR ALL-

NEG. FOR FIXED RELATIONSHIP. END WITH 0.

User: 99 designates all peaks in the list as free in the fitting process. 
nnn (sequence number) designates the centroid position of the peak as variable in the fitting process. Those not designated in this step are fixed in position to the channel corresponding to the energy of the peak entered, by the formula

$$
\text { peak position }=(\text { peak energy }- \text { zero channel energy }) / \text { gain }
$$

-nnn (negative sequence number) fixes the peak energy relative to another peak in the region. The sequence number of this other peak in the form nnn is entered in response to the prompt.

\section{ENERGY FIXED RELATIVE TO PEAK \#:}

The centroid of the current peak is then varied as the centroid of the peak to which it has been fixed. This option is used for splitting close multiplets or for locating weak peaks.

t terminates the entry of sequence numbers of peaks of uncertain energy.

\section{Routine: SEQUENCE \# OF PEAK WHOSE INTENSITY IS KNOWN}

User: nnn (sequence number) indicates that the intensity of this peak is known. A zero entry indicates that all are unknown. The peak height is entered after the prompt.

PEAK HEIGHT (NEG. FOR RATIO) =

$x \operatorname{xx} x \times x \times . x x x x$

designates the peak height for the sequence-numbered peak named above.

$-x x x x x x x x . x \times x x$

(negative entry) specifies that the peak height is to be ratioed to another peak in the region, a procedure useful when both peaks are from the same isotope and their branching ratios are well known. The absolute value of this entry is the ratio of the current peak height to the height of the reference peak.

Enter the sequence number of the reference peak in the form nnn following the prompt RATIOED TO PEAK \#: .

\section{Routine: IS THERE A PARAMETER FILE?}

User: $\quad Y \quad$ reads in a file of peak-shape parameters available on disk. Enter the file name in the form device.file after the prompt FILE NAME?

After the file name is entered, the routine asks

DO YOU WANT TO FREE ANY SHAPE PARAMETERS? 
$Y$ frees this shape parameter. The maximum number of free parameters, including peak positions and beights. that are allowed for fitting each peak grouping is 20 .

N fixes the parameter to the value determined from the parameter file, in accordance with Eqs. (3) and (4), above.

$\mathrm{N}$ indicates there is no parameter file. If there is none. enter a value for each peak-shape parameter, as listed below. For each, a response to the prompt TO BE FREED? is required.

Y frees the parameter.

N fixes the parameter to the value entered.

The peak-shape parameters that can be freed for the entire peak grouping are:

FWHM (IN KEV)
SHORT-TERM TAIL AMPLITUDE
SHORT-TERM TAILING SLOPE
LONG-TERM TAIL AMPLITUDE
LONG-TERM TAIL SLOPE

The height and the position may be freed for each peak in the multiplet. 4 20-peak multiplet may be fit if peak height is the only free parameter. A seven-peak multiplet can be fit if all parameters are free.

\section{Routine: LOW ENERGY BKGRD SLOPE EXPRESSED IN \%/CHAN =}

User: $\quad x \cdot x x x \quad$ specifies the value for the slope before the region of interest, expressed as $\%$ change in counts per channel relative to the average counts per channel in the region. This option is useful for peaks which lie in a region where the Compton continuum is not llat, but has a nonzero slope.

Routine: HIGH ENERGY BKGRD SLOPE EXPRESSED IN \%/CHAN =

User: $\quad x \cdot x x x \quad$ specifies a similar value for the slope following the region of interest. 


\section{REFERENCES}

1. 3. E. Fager and F. P. Brauer, Rapid Nondestructive Plutonium Jsotopic Analysis, U.S. National Burealu of Standards Special Publication 528, 64 (1978).

2. Currently used at Mcund L/aboratories. Miamisburg, Ohio.

3. R. Gunnink and J. B. Nday. Computerized Quantitative Analysis by Gamma-Ray Spectrometer. Lawrence Livermore Natisinal Laboratory, Livermore, Calif., UCRL-57061 (1971).

4. R. Gunnink. Computer Techniques for Analysis of Gamma-Ray Spectra. Lawrence Livermore National Laboratory. Livermore, Calif., UCRL-80297 (1978).

5. J. J. Routti and S. G. Prussin, "Photopeak Method for the Computer Analysis of Gammi-Ray Spectrat from Semiconductor Detectors," Nucl. Inst. and Methods 72, 125 (1969).

6. G. Phillips and K. W, M srlow, "A Atomatic Analysis of Gamma-Ray Spectra from Germanium Detectors." Nucl. Inst. and Methods 137. 525 (1976).

7. H. P. Block et al. “A New Peak Search Method for an Automatic Spectrum Analysis System." viucl. Insi. and Methods 128, 545 (1975).

8. W. M. Sanders and D. M. Holmes, An Analytical Method for Unfolding Gamma-Ray Spectra, Los Alamos Scientific Laboratory, Los Alamos, N.M., LA-4038 (1969).

9. R. Gunnink. “An Algorithm for Fitting Lorentzian-Broadened K-Series X-Ray Peaks of the Heavy Elements." Nucl. Inst. and Methods 143. 145 (1977).

10. R. Gunnink and J. B. Niday, in: ERDA Symposium on X-and Gamma-Ray Sources and Applications (U. S. Energy Research and Development Administration. CONF-760539, Washington. D.C.. GPO. 1976) p. 55.

11. S. I. Salom and P. L. Lee, "Experimental Widths of K and L X-Ray Lines," Atomic Data and Nuclear Data Tables 18(3) 233 (1976). 


\title{
APPENDIX: SOURCE LISTINGS
}

\author{
GRPANL \\ ULOAD2 \\ ULFIT2 \\ ULOUT2 \\ FILES \\ PKSHAP
}

\section{Subroutines:}

\author{
RDSK \\ YESNO \\ MAXVAL \\ MINVAL \\ NCNTS \\ NCTS \\ AVE \\ BKGRD \\ GFIT \\ BWF \\ MLR \\ MLRA \\ MLRE \\ GPM \\ CALMU \\ CALGPM
}


PRIGGFAM GRFAANL

E

C.

LINK WI TH FIISK, YESNO, AUE, HAXUAL, GFIT, NCNTS, NCTS

THIS FROGFAM IS A GENERAL FURPOSE FFOGRAM FOK FITTING MULTIFIETS (GAMMA-FAYS ANII $X$-FAYS).

IT WILL ACCOMMOIATE UF TO 20 FREE FARAMETERS. THE PEAK HEIGHTS (AKEAS) AFE AUTOMATICALLY FREEL. THE OTHERS CAN FE SELECTEI, UF TD 240 IIATA FOINTS AFIE ALLDWEI.

0001

000

onus

1004

9045

c

0006

0007

oope

0009

0010

0011

0012

0013

0015

0016

0017

0018

.0019

0020

0021

0022

0023

0024

0025

0026

0027

0028

0029

0030

0031

0032

0033

0034

0035

0036

0037

0038

0039

C

C

THIS FROGEAM REALIS IN GRFANL F'ARAMETEFS

FFOIY TTY TFFMINAL

\section{INTEGEF*2 LLLDAII2(4)}

LDGIEAL YESNO

IIIMENSIGN AMATHX(58BE), BMATFX(256)

COHMON / GNL/NLIMS, NUME, NIFF S NF, NWFIS $(6)$, NF L.G (2O), NYFLG (20),

1 NXFLE (20), FNAME (3), SF (2), GAIN, ZEFO, GTAFT, ALFHA, FWHW, ATAIL .

2 ETAIL,EXF', EXF'4, STH(2), C1, C2, AVEEG, SLF (2), EN(20), SHAFC(10),

3 CLIN(5), HIGHT $(20)$, GAHA $(20), Y(240),[1 U H(1500)$

COMMON/FASS/EN J (2O) , IST, IENI, NUMS J, NUHE J, GAIN J, ZEROJ, S I 1 J,

1 ST2J, STFU 20$)$, NENUM, ALF J, STAHF, STSLF, TLAMF, TLSLF, EKGSLF (2),

2 CFILE ( 3 ) , 5SS, YES, ERI 1 , NGAIN, IFEGG, L I STFG, FAME (3)

EQUIUALENCE (NUMS, AMATFX (1), (EN I 1 ), EMATFX(1))

C LISTFG = LISTING FLAG = O COMFLETE FFINTOUT

C. JF CALCULATIONS

C = 1 SHOFT FRINTOUT

C THE FOLLOWING STATEKENT FROUIIES FOF CHAINING TO THE FFOGKAM

C ULOAII2 ON LQGICAL IIEUICE RK3. IT MUST FE CHANGEI ACCOFIINGLY

C IF THE FFOGFiAM FIESIDES ELSEWHEFE.

IIATA ULOAII2/3RKK3, 3RULO, 3FAD2, 3KGAU/

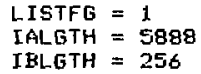

106 FORIMAT (I4)

SUM(J) $=0$.

AMF1 $1=$ EXF'1

AMF2 = EXF3

GANMA $=0$.

$X I=F K F O S(N)$

I0 $300 \mathrm{~L}=1,2$

CC $=\mathrm{CX} * \mathrm{XI}$

IF (GAMA ( J) ) 201,201,100

100 CALL BWF (CC)

201 BG(L)=EG(L)+FKHT $(J) *(.6366 * G A M A(J) * C X * C C+A M F 1 * E X F(-E X P 2 * X I)$

1 TAMF2 2 EXF(-EXF4*XI) 


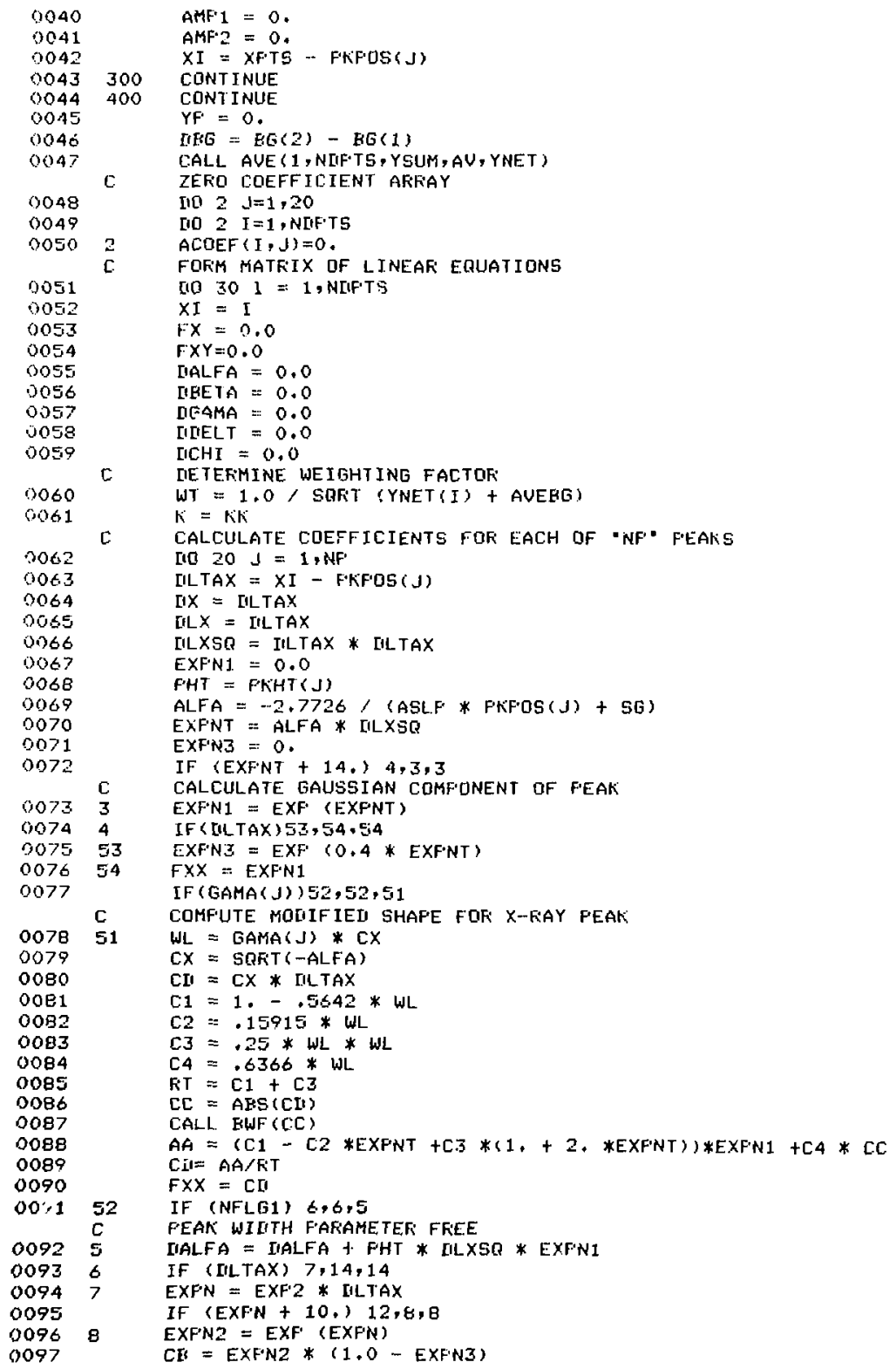




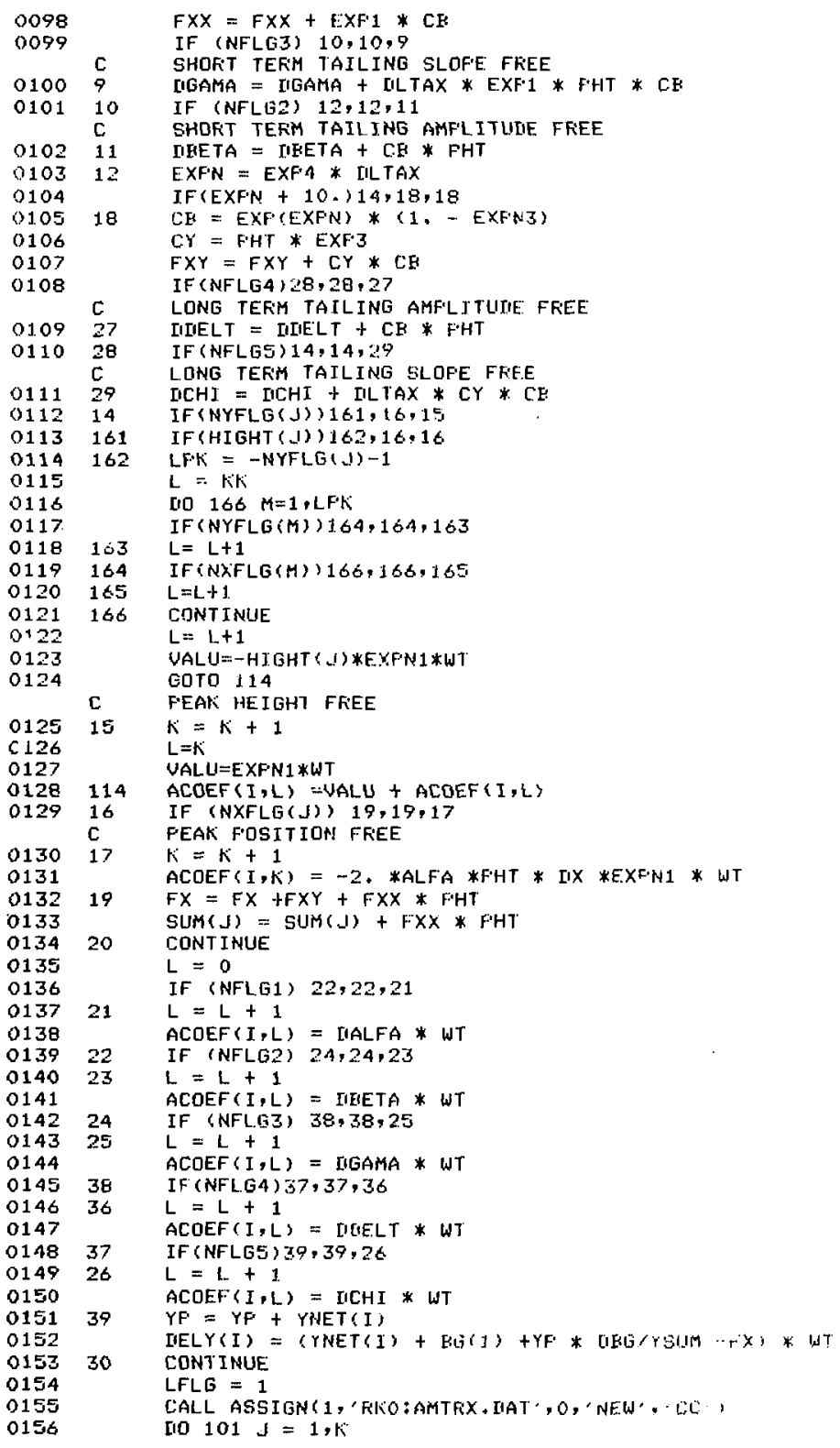




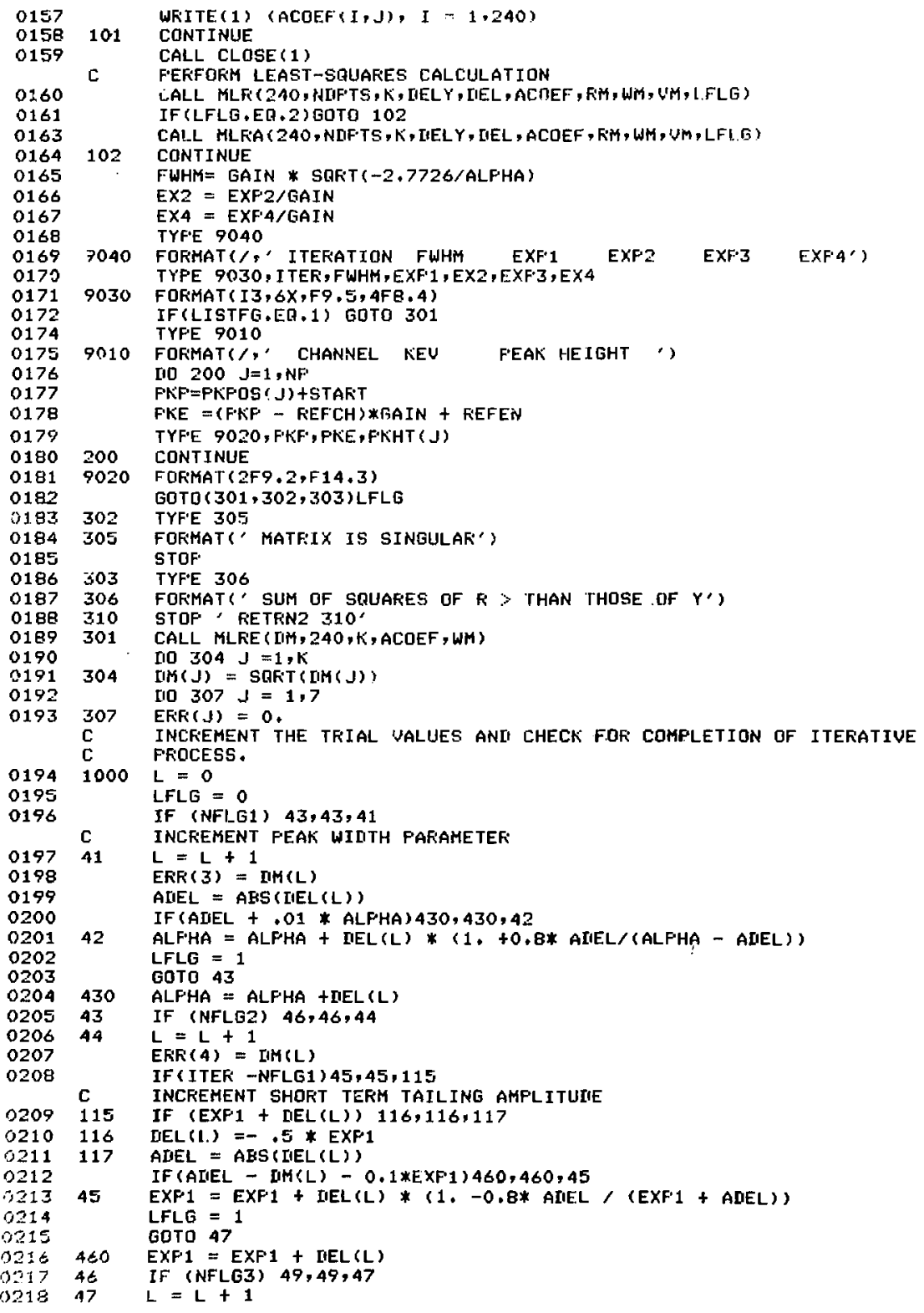




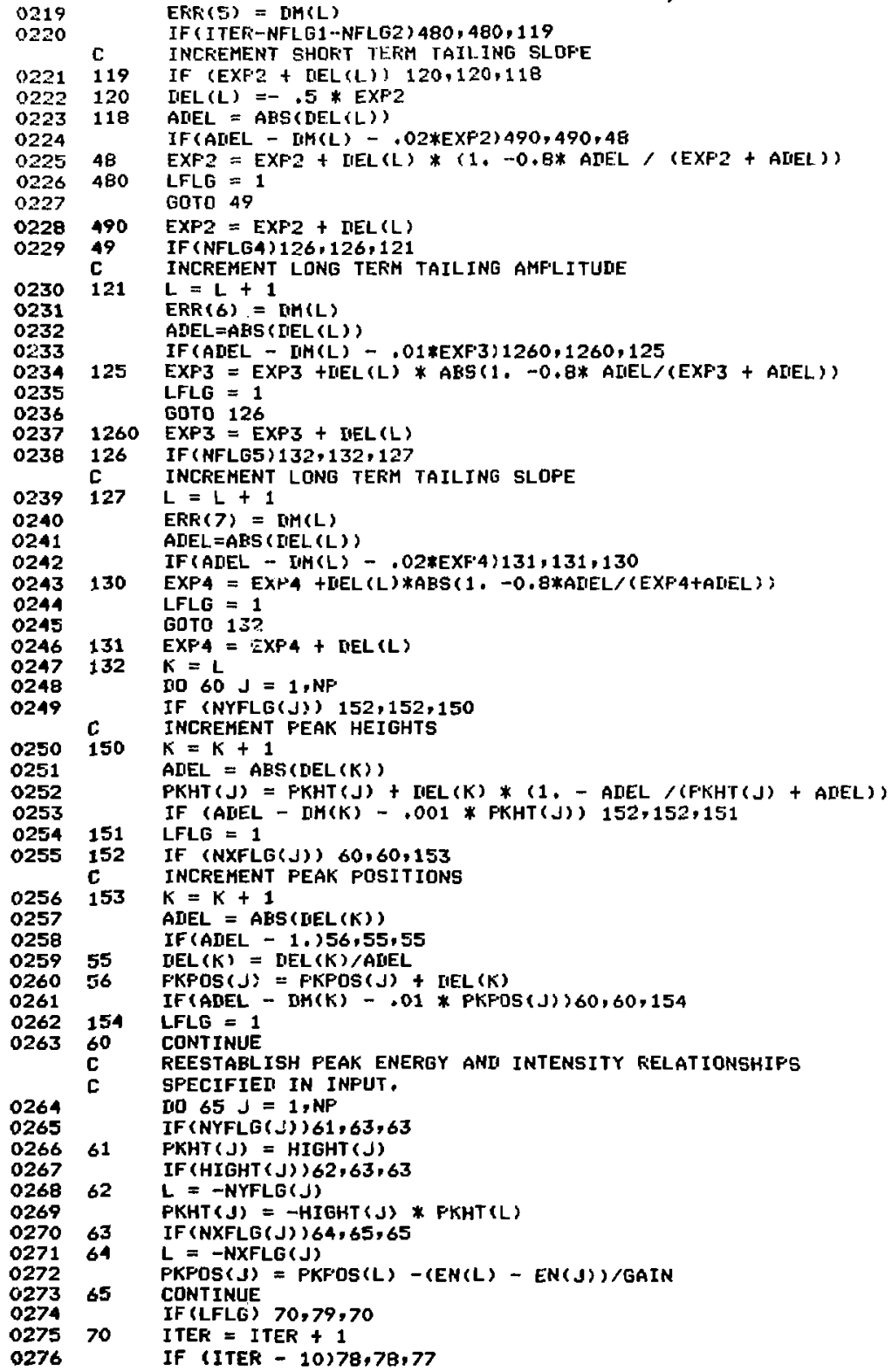


C ITERATE CALCULATIOH agaIH

027779 Da $901=1.5$

0279 IF (NFLG(I))91,90,90

$027991 \quad$ NFLG(I) $=$ - NFLG(I)

O2BC 70 CONTINUE

0281 GOTO 1

028277 TYFE 105

0283105 FUKHAT ('MAXIMUH ITEKATIOHS EXCEELED.')

СССССССССССССССССССССССССССССССССССССС СССССССССССССССССССС

C TERHINATE ITERATIOHS. QUTFUT FESULTS.

028479 CFLL ASSISN(NIEU,'SY:GNL. HAT')

0285

0286

0287

0288

0289

0290

0291

WRITE (NUEU) (AHATFX(I), I = I, IALGTH)

CALL CLOEE (NUEV)

CAL! ASSIGMCHUEU,'SY:FASS, BAT':

WFITE (WIEU) (EHATFX(I), I $=1, I Z L G T H$ )

CALL CLOSE (NIEV)

CALL CHATN(ULOUT2,0,0)

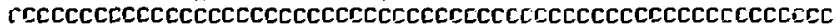
ENI 


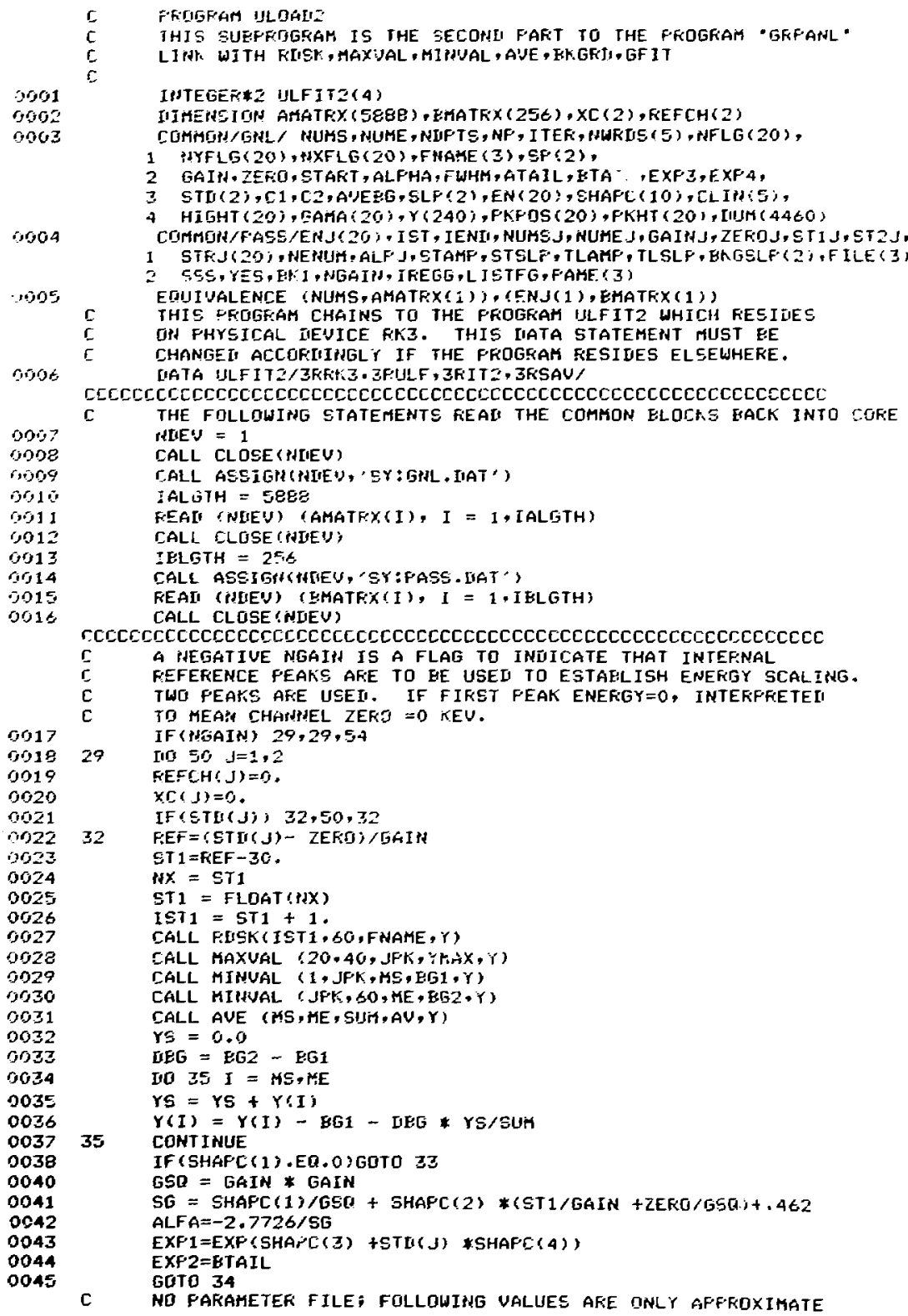




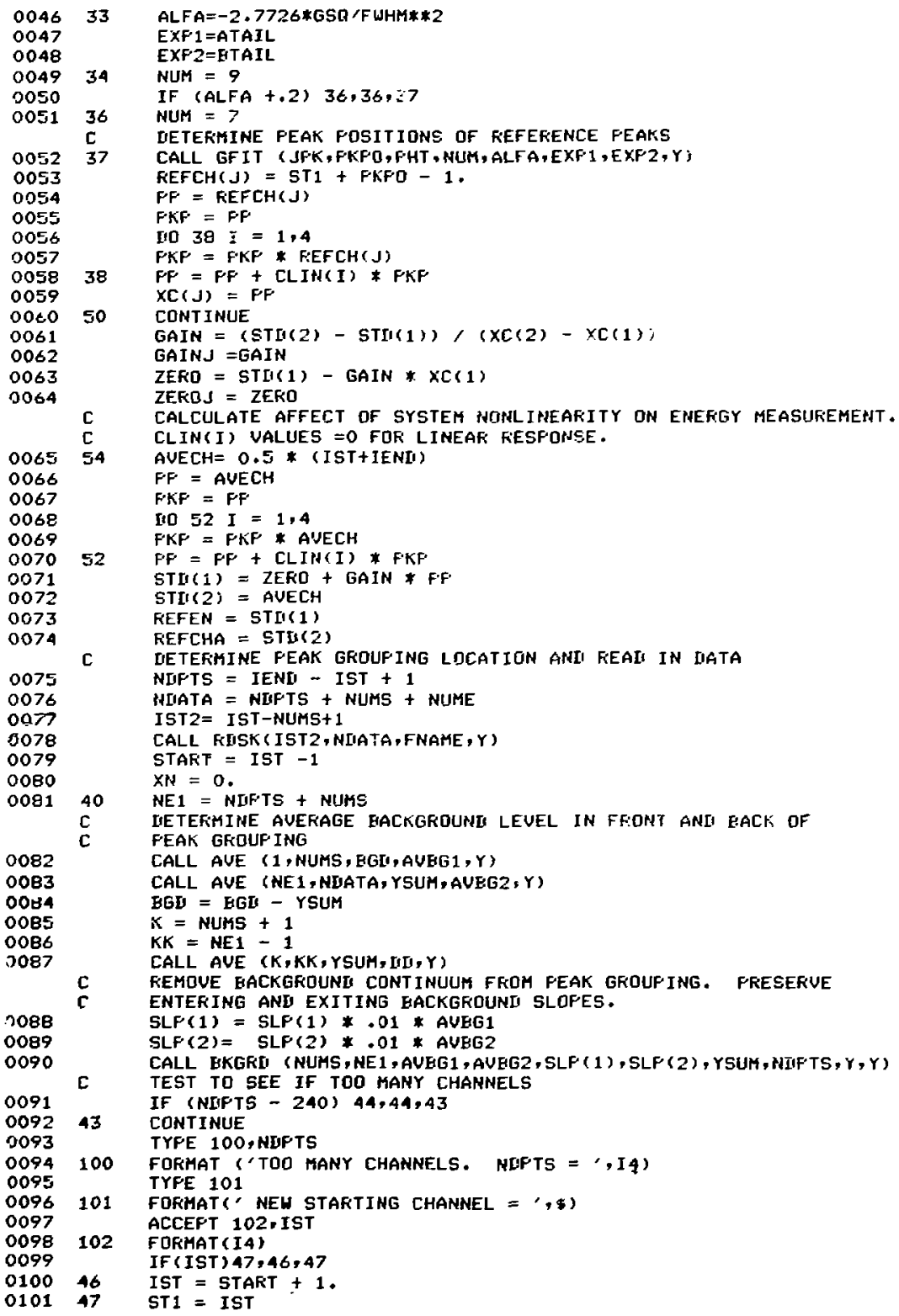




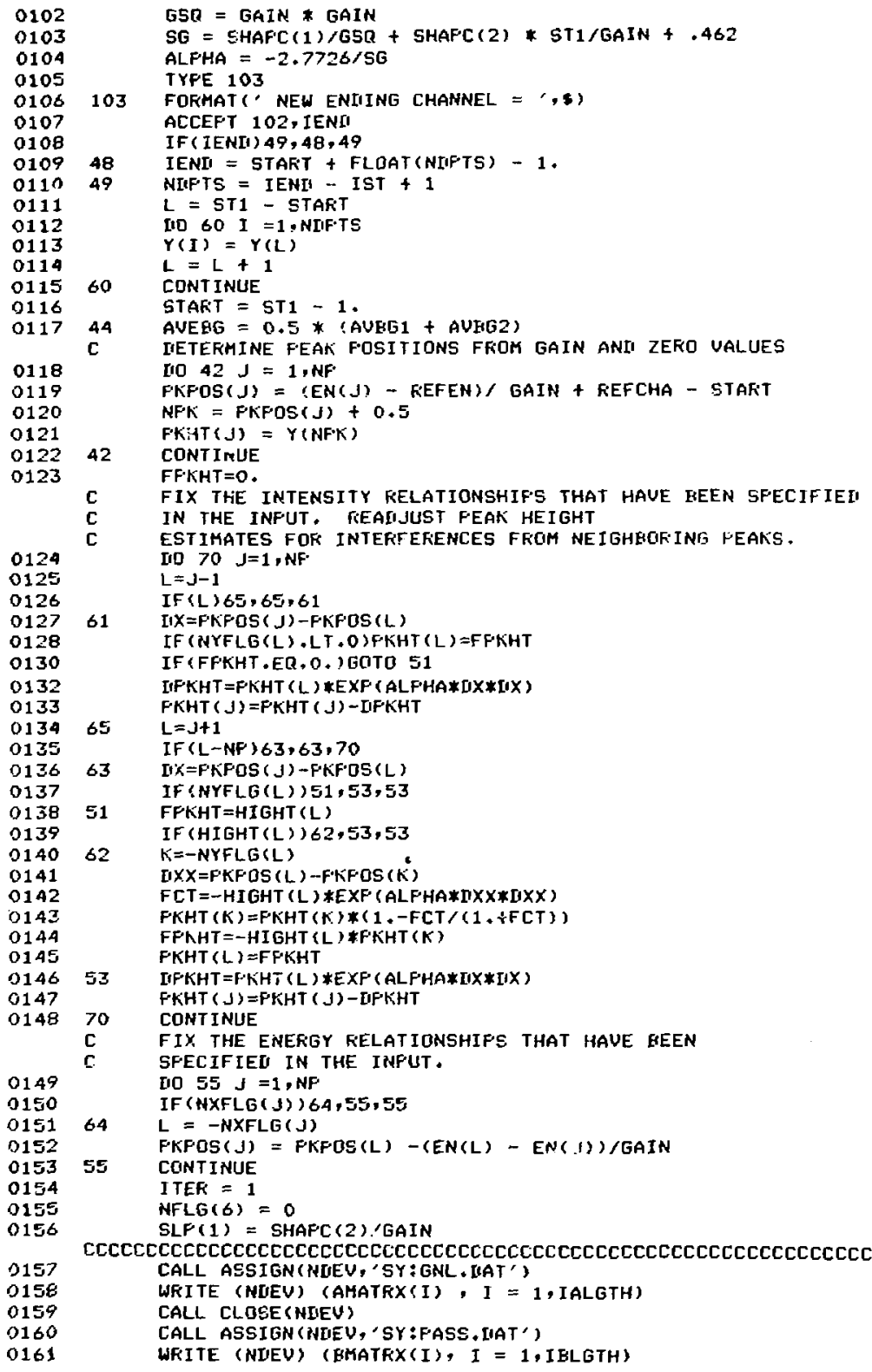


CALL CLOSE (NUEU)

0164

CALL CHAIN(ULFIT2,0,0)

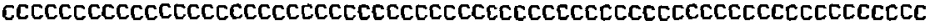
ENII 


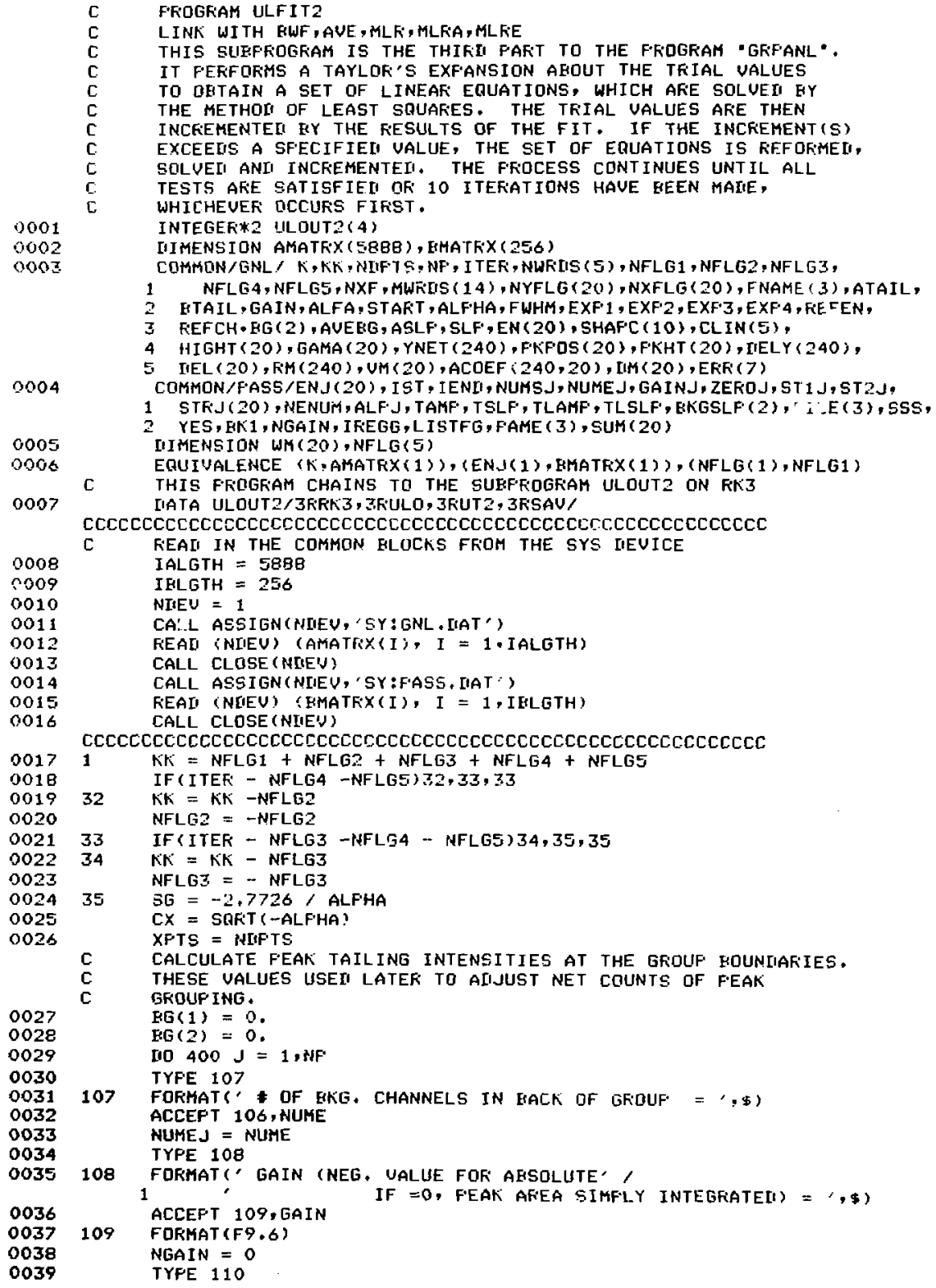




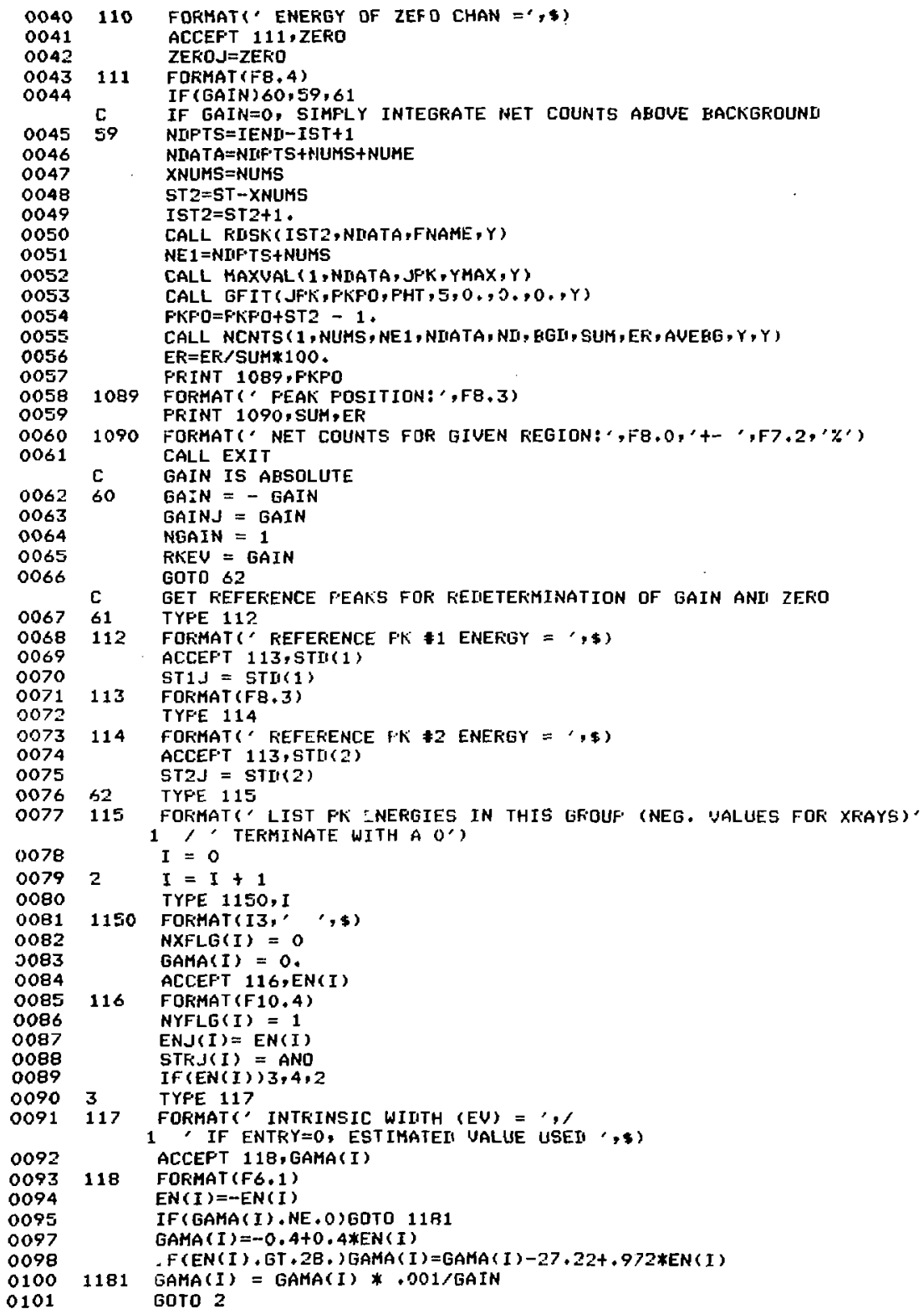




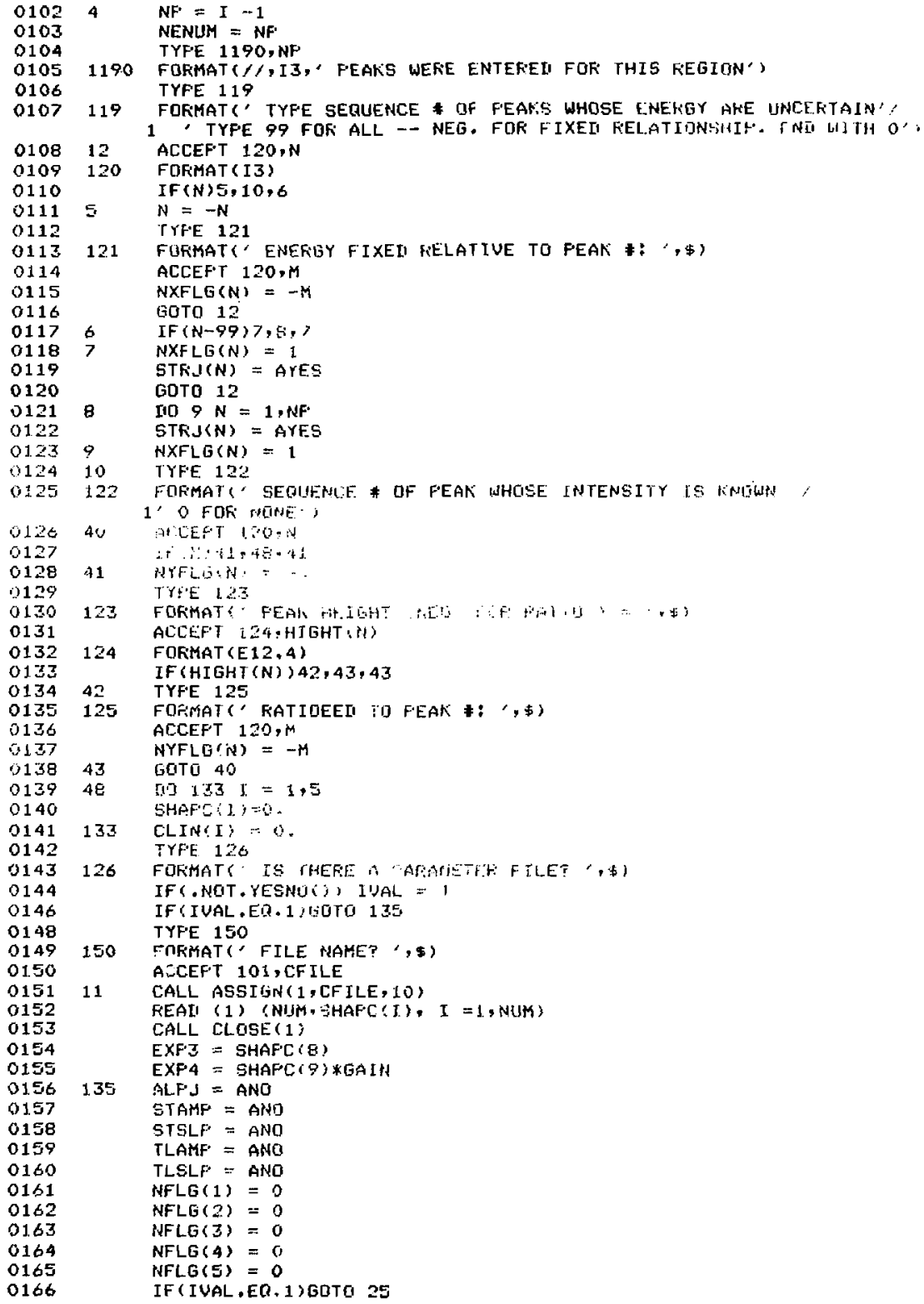




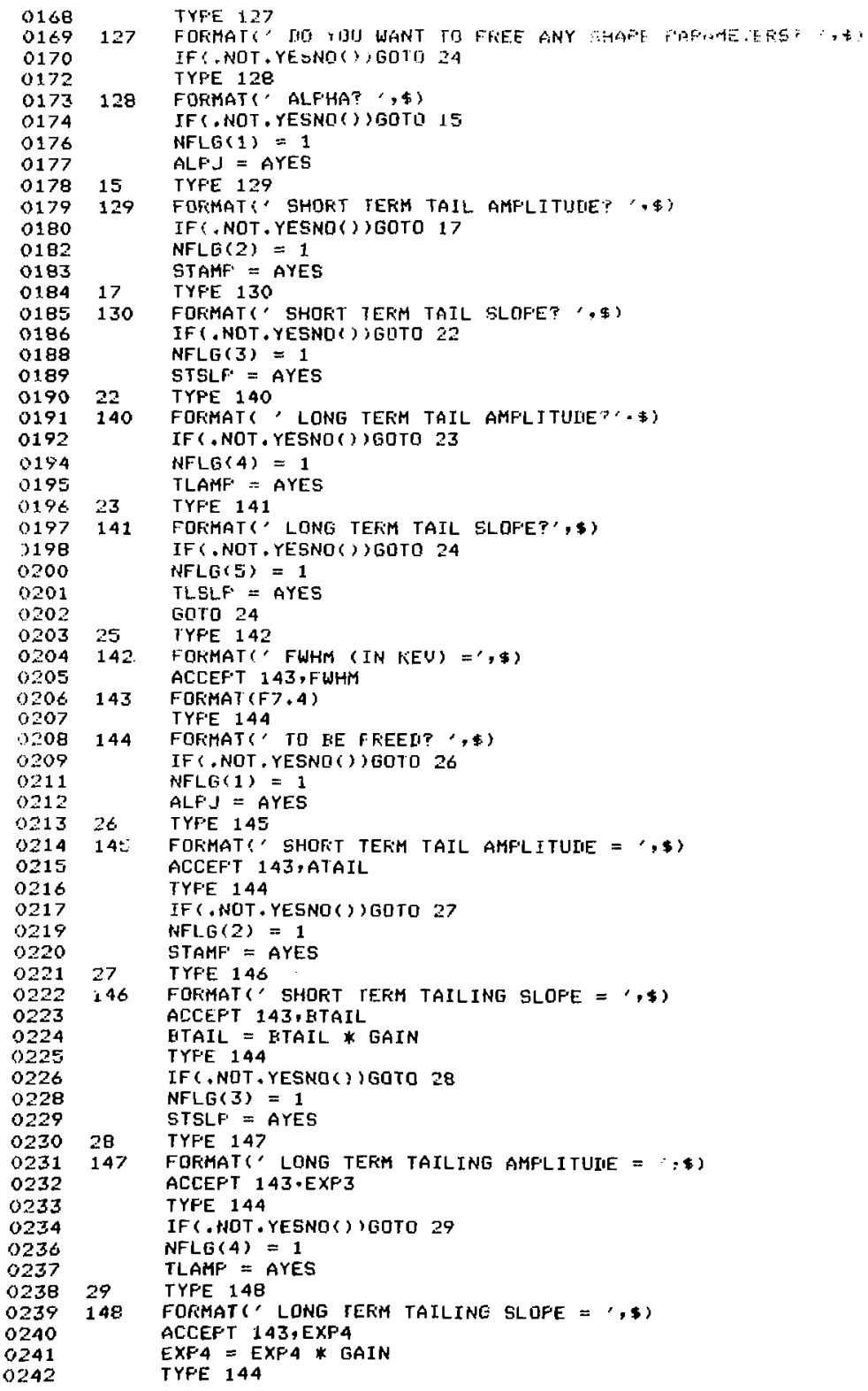




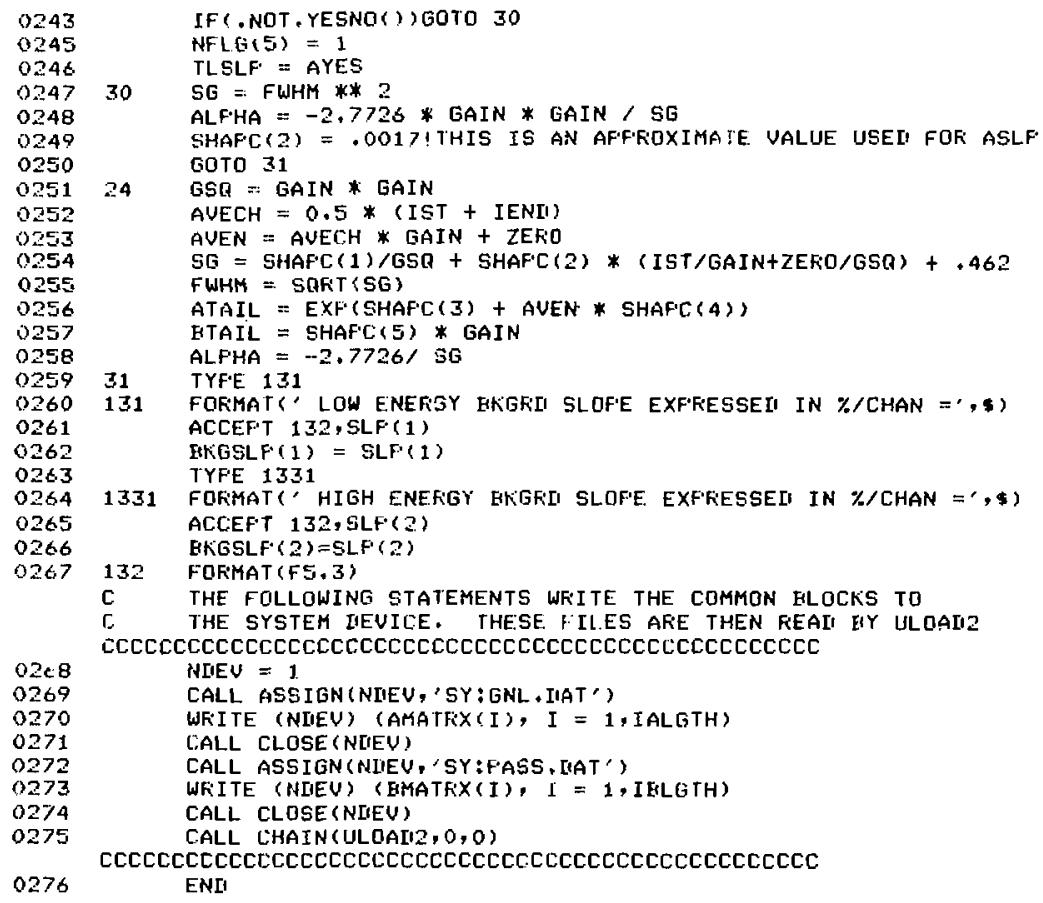




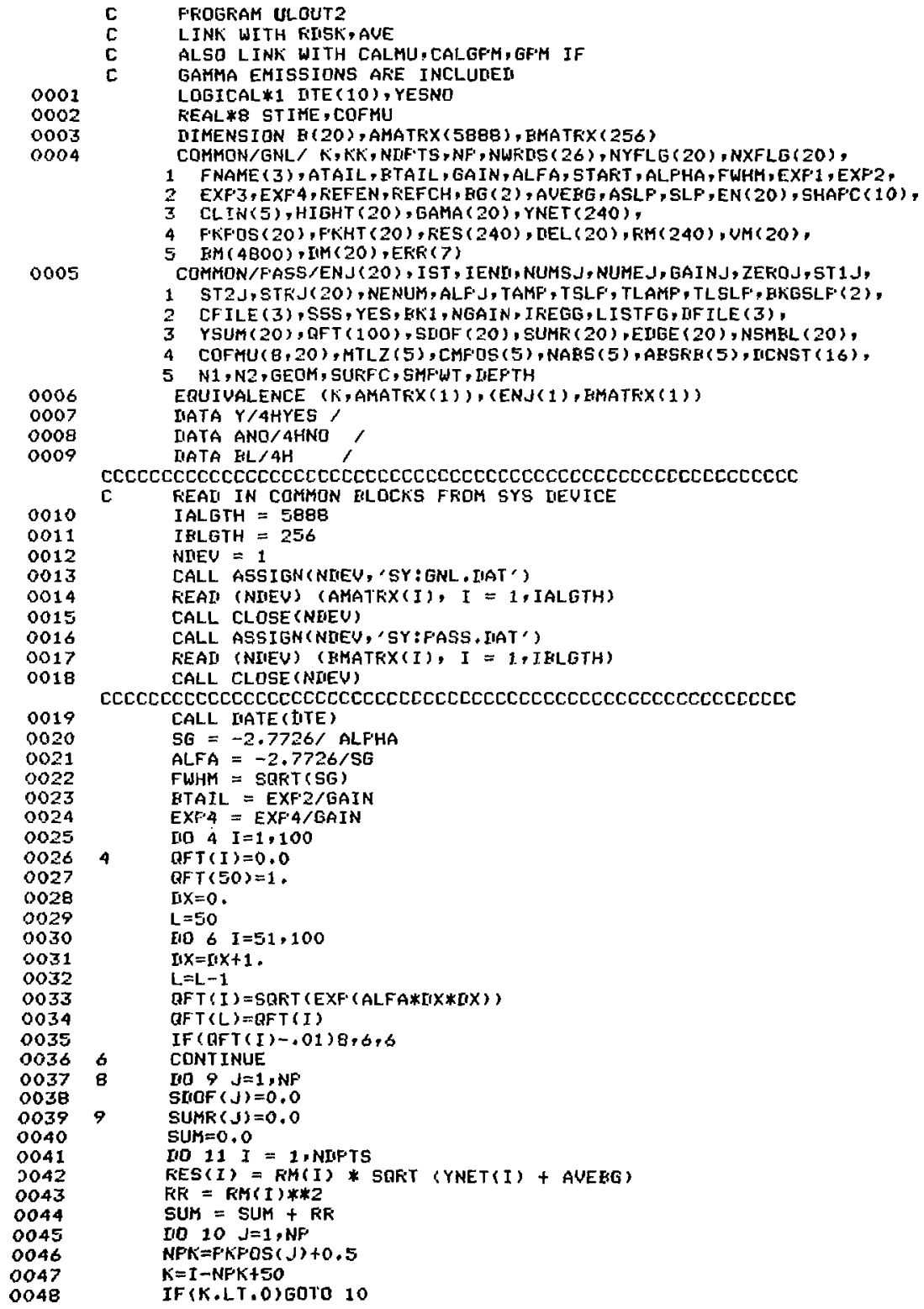




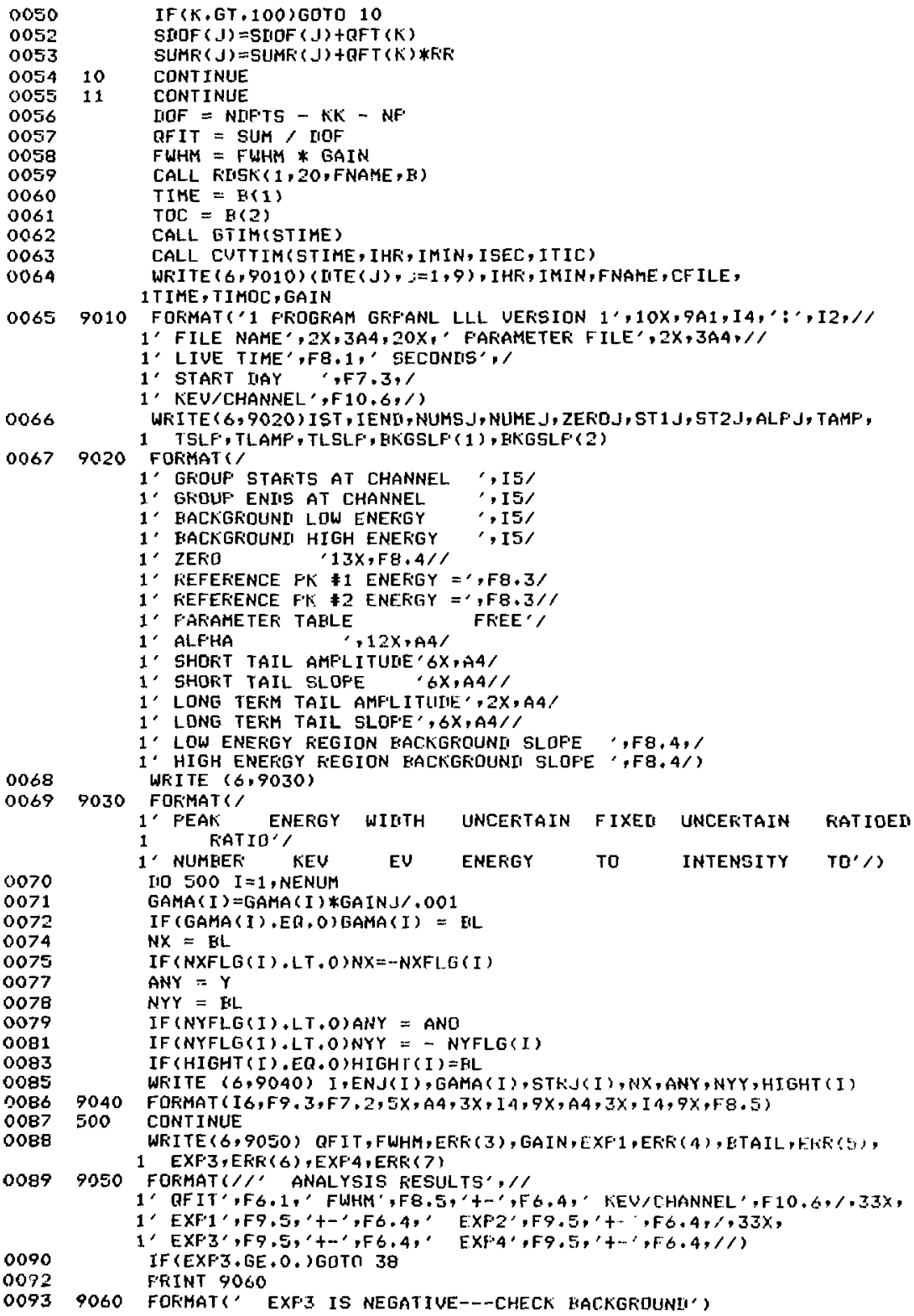




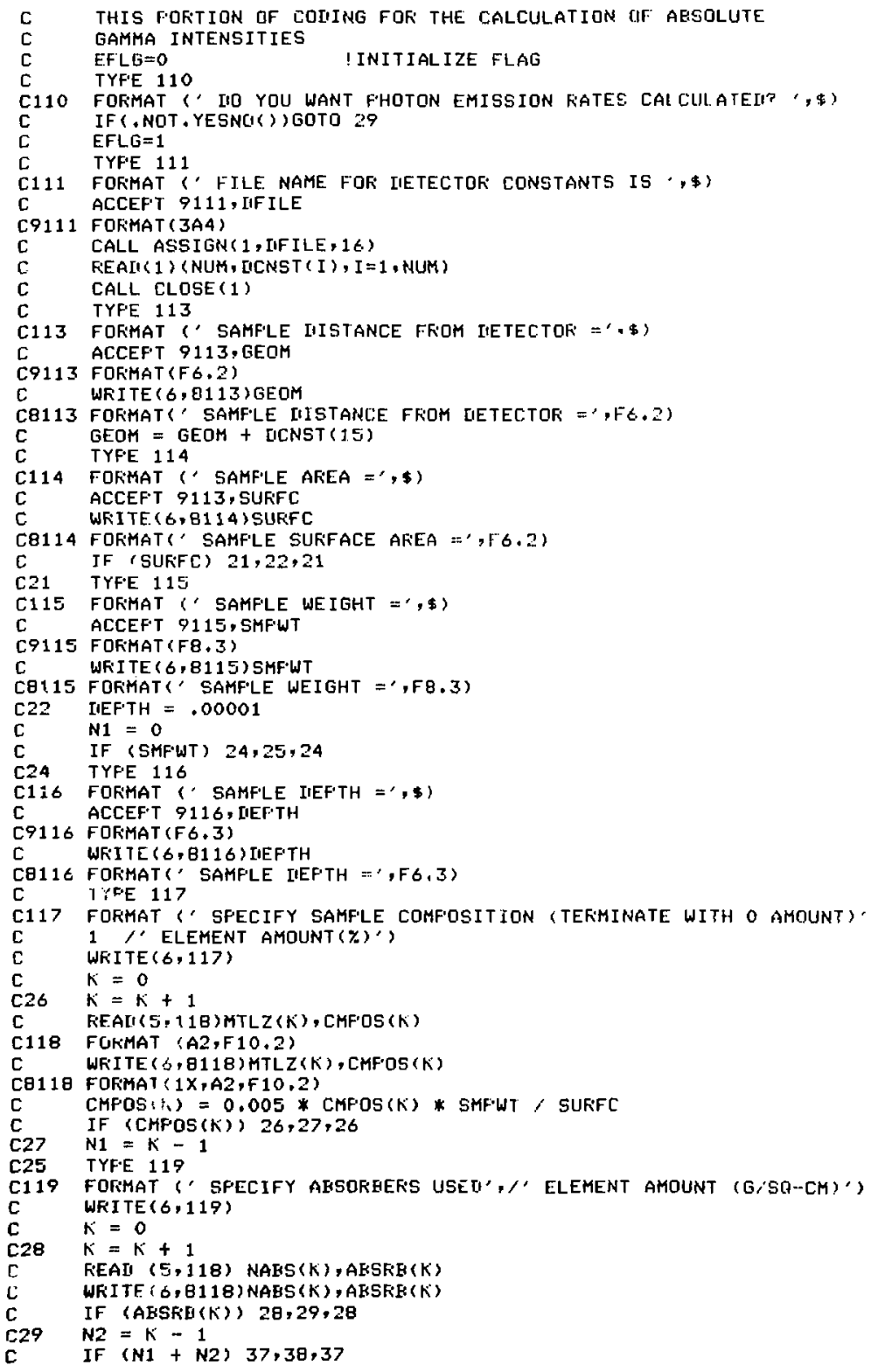




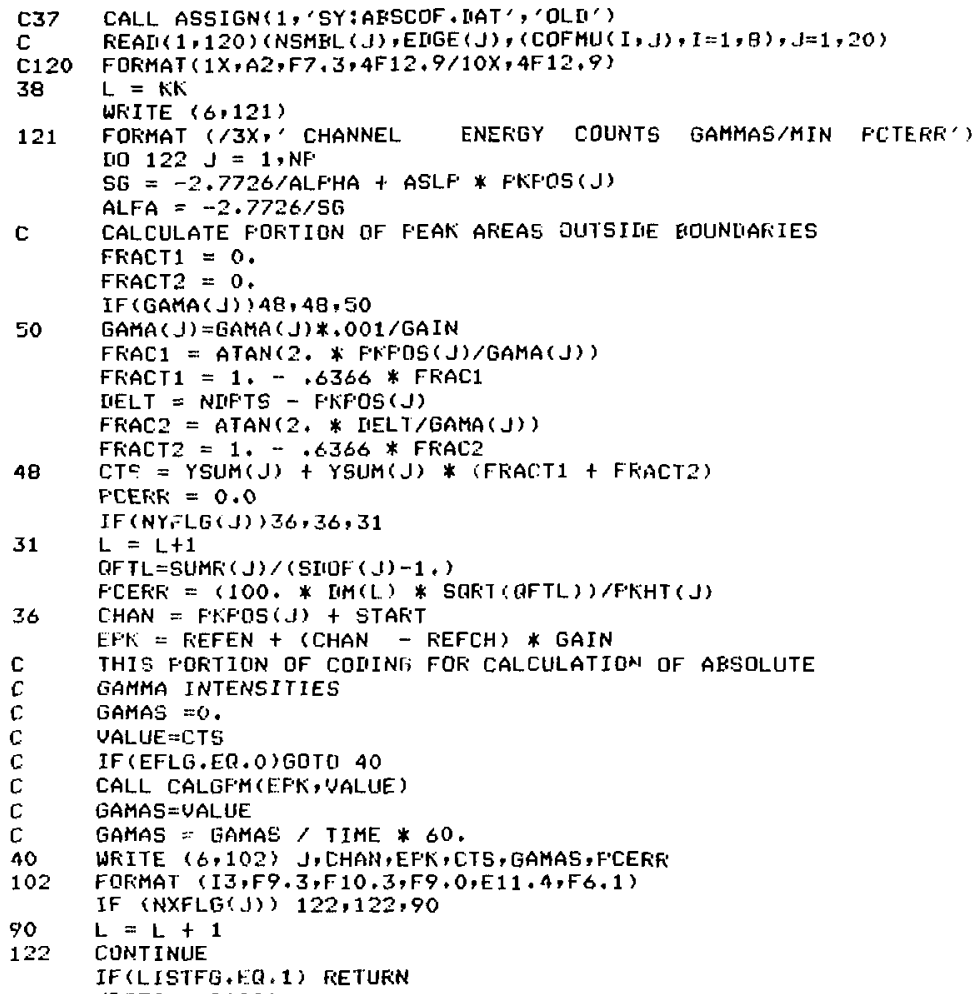




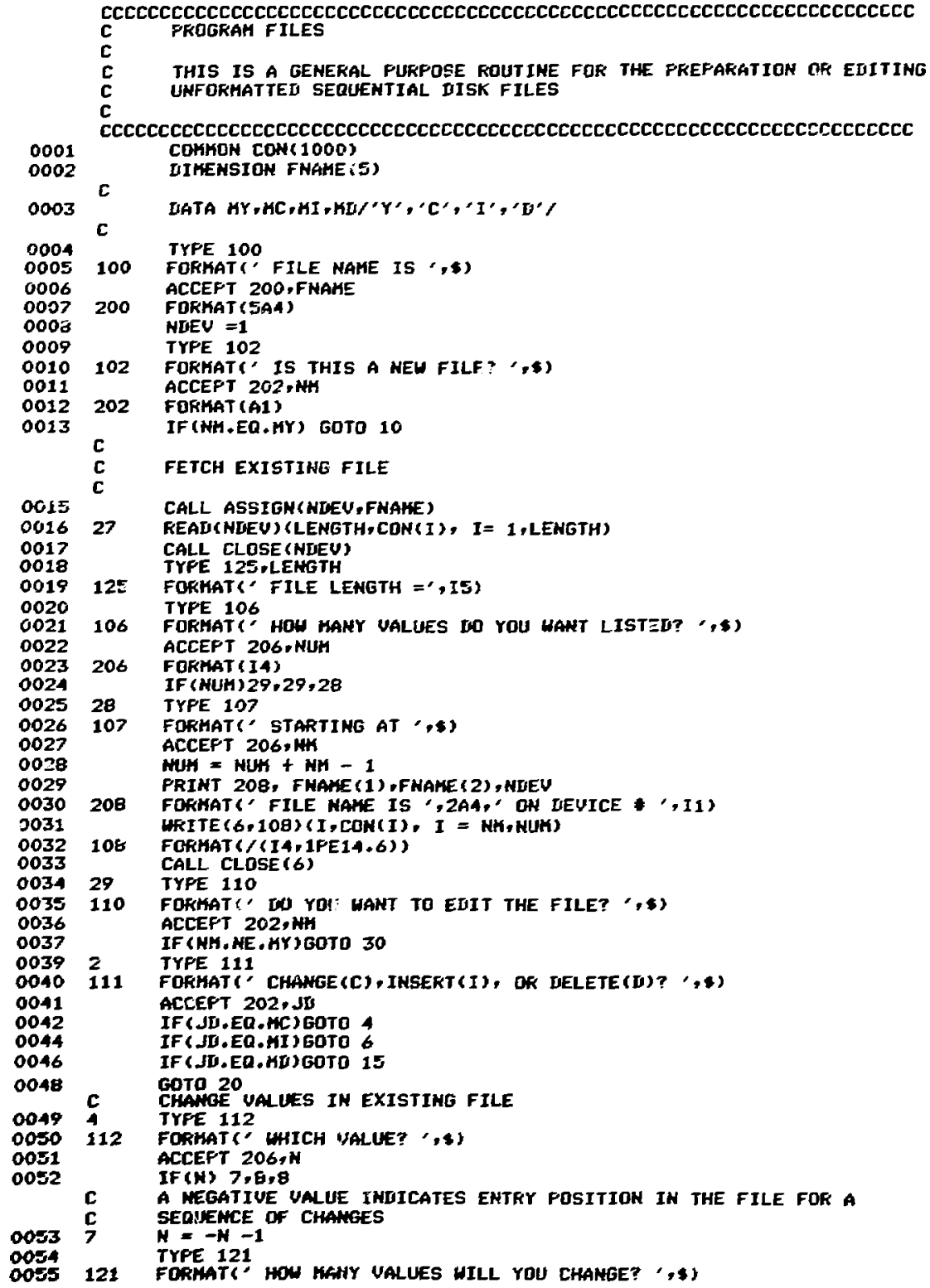




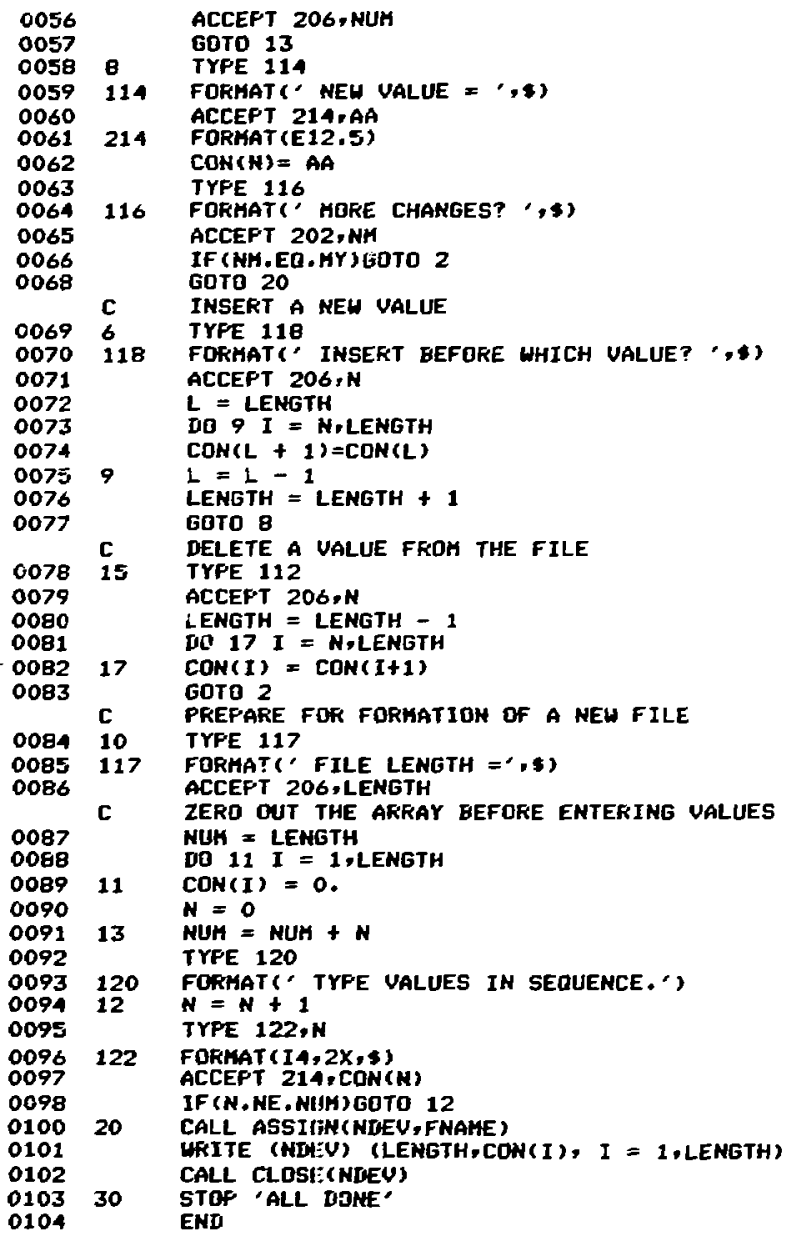




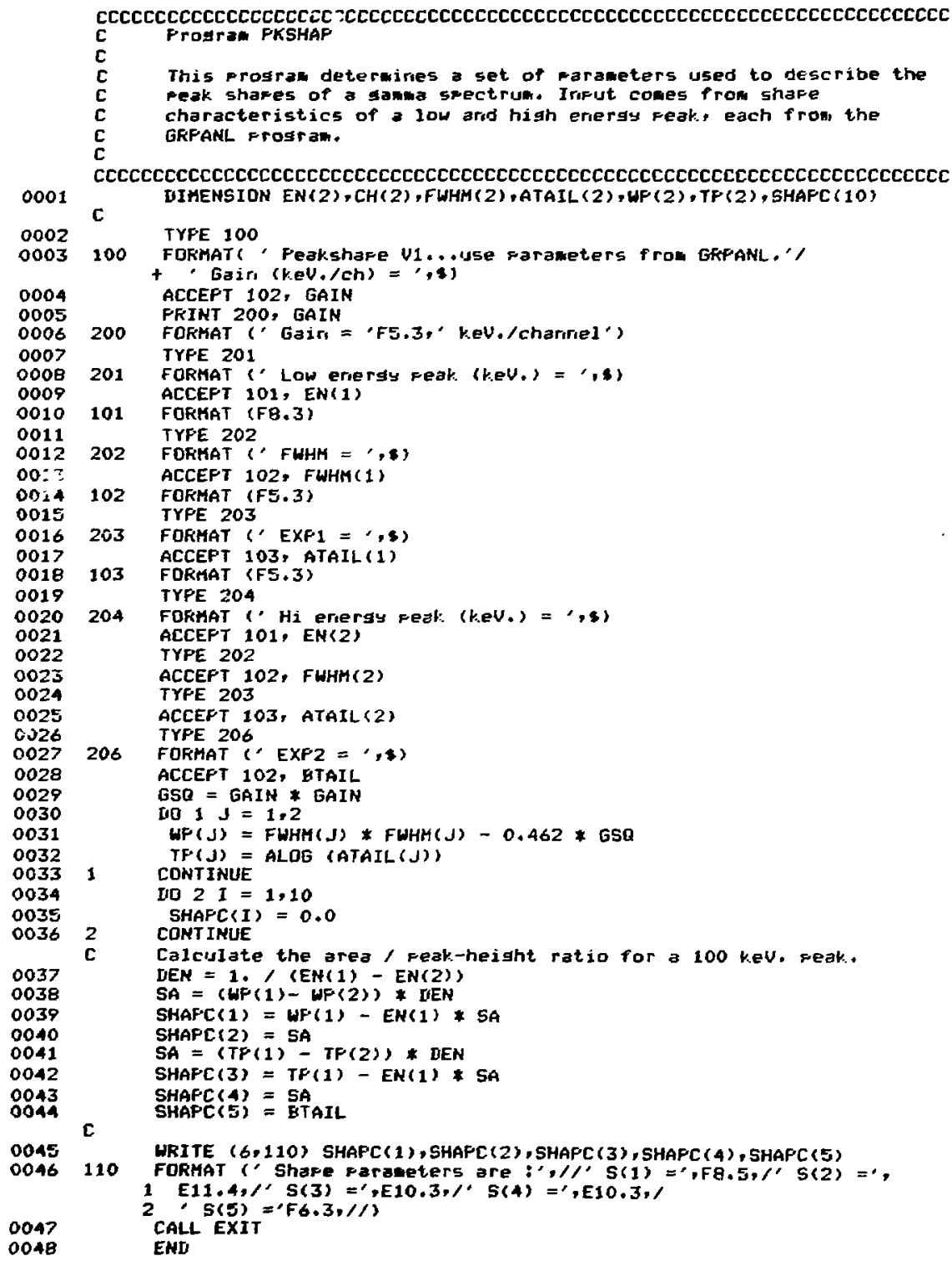




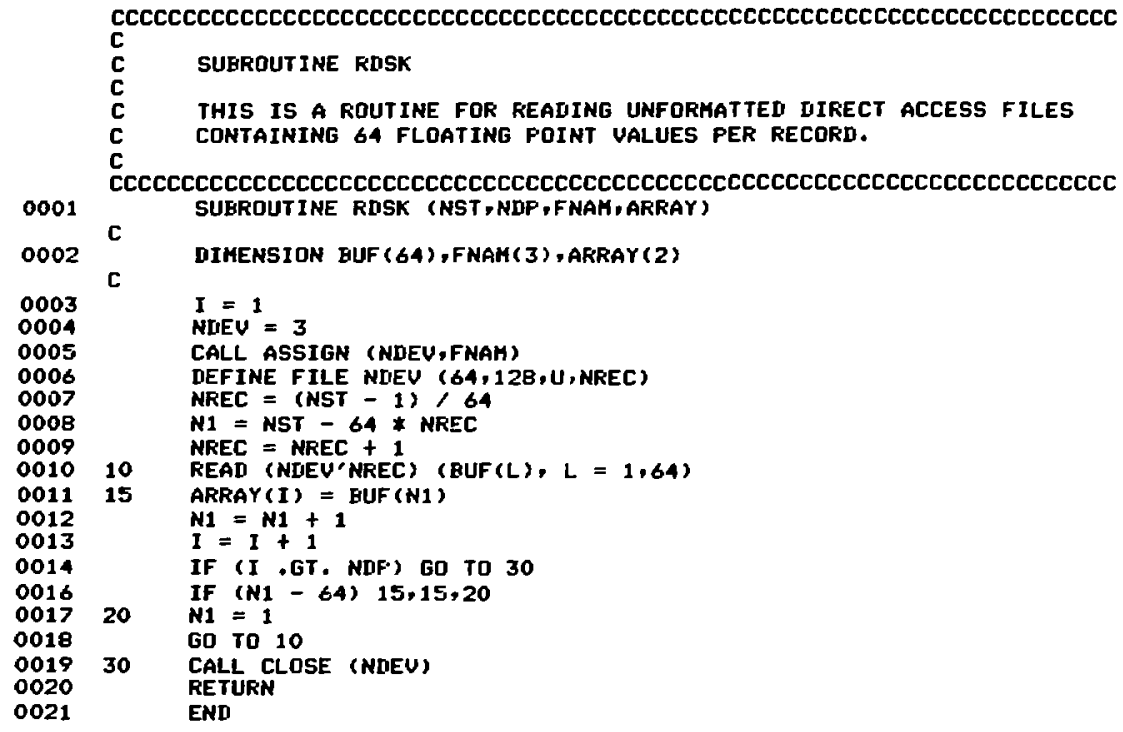




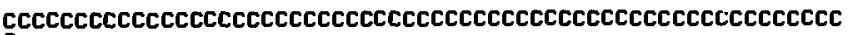

C LOGICAL FUMCTION YESNO

C

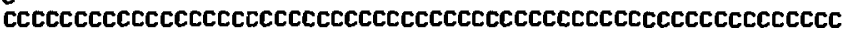

c

C

0001

0002

0003

0004

0006

0007

0008

0010

0011

0013

0014

0015

0016

0017

0018

0019

0020

LOGICAL FUNCTION YESNDFI (IANS)

LOGICAL $* 1$ IST (6), ISTR (4)

$\mathbf{C}$

CALL GETSTR $(5$, IST, 3,ERR)

IF (ERR . EQ. . TRUE. ) GD TO 10

CALL SUBSTR(IST, ISTR,1,1)

CALL SCOHP(' $Y$ ', ISTR, IUAL')

IF (IUAL.ER. 0 ) GO TO 20

CALL SCOMP('N', ISTR,IUAL)

IF (IUAL.ER. 0) GD TD 30

10 TYPE 100

100 FORMAT(" PLEASE ANSUER Y DK $\mathrm{N}^{\prime}$ )

GOTO 5

20 YESNO $=$. TRUE.

C

30 YESNO = .FALSE.

RETURM

END 


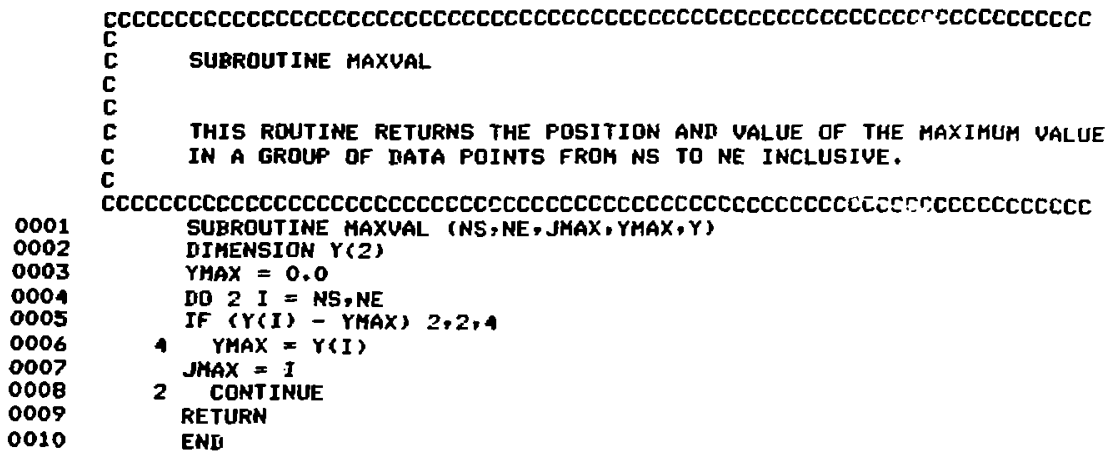

C

C

C THIS ROUTIME RETURNS THE POSITION AND VALUE of THE MaXIMUM VALUE 


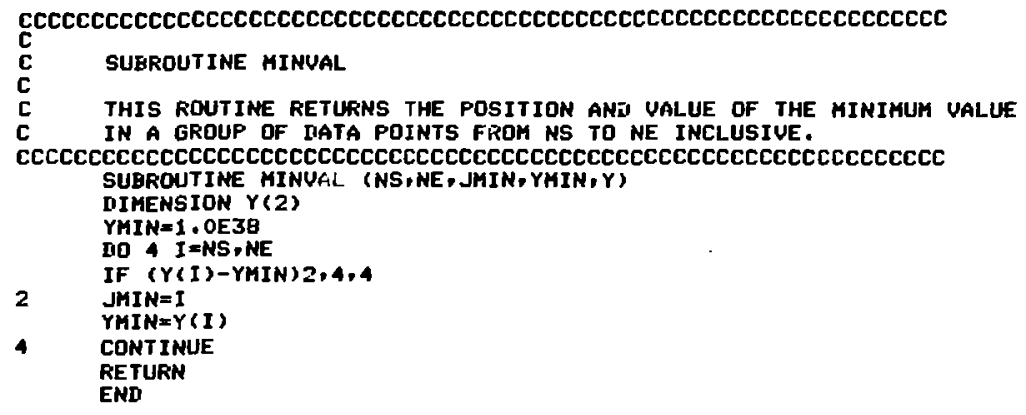




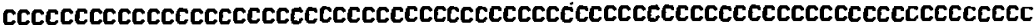
Subroutine P'n'ts

C This routine is siailar to NCTS excert it returns on averase

C backsround and an error associated with the total net courit.

C

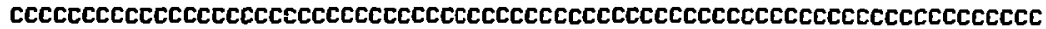

0001

0002

0003

0004

0005

0006

0007

0009

0010

0011 SUBROUTINE NCNTS (N1,N2, H1, H2, NI,S1,S2,ER, AUEBG,Y1, Y2)

C DIMENSION $Y 1(2), Y 2(2)$

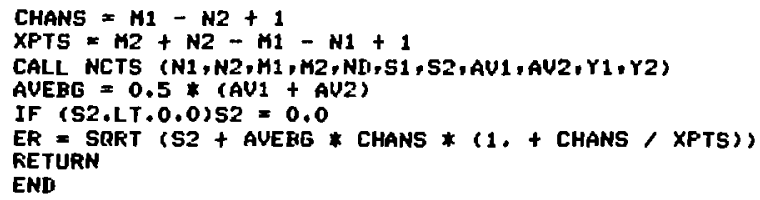




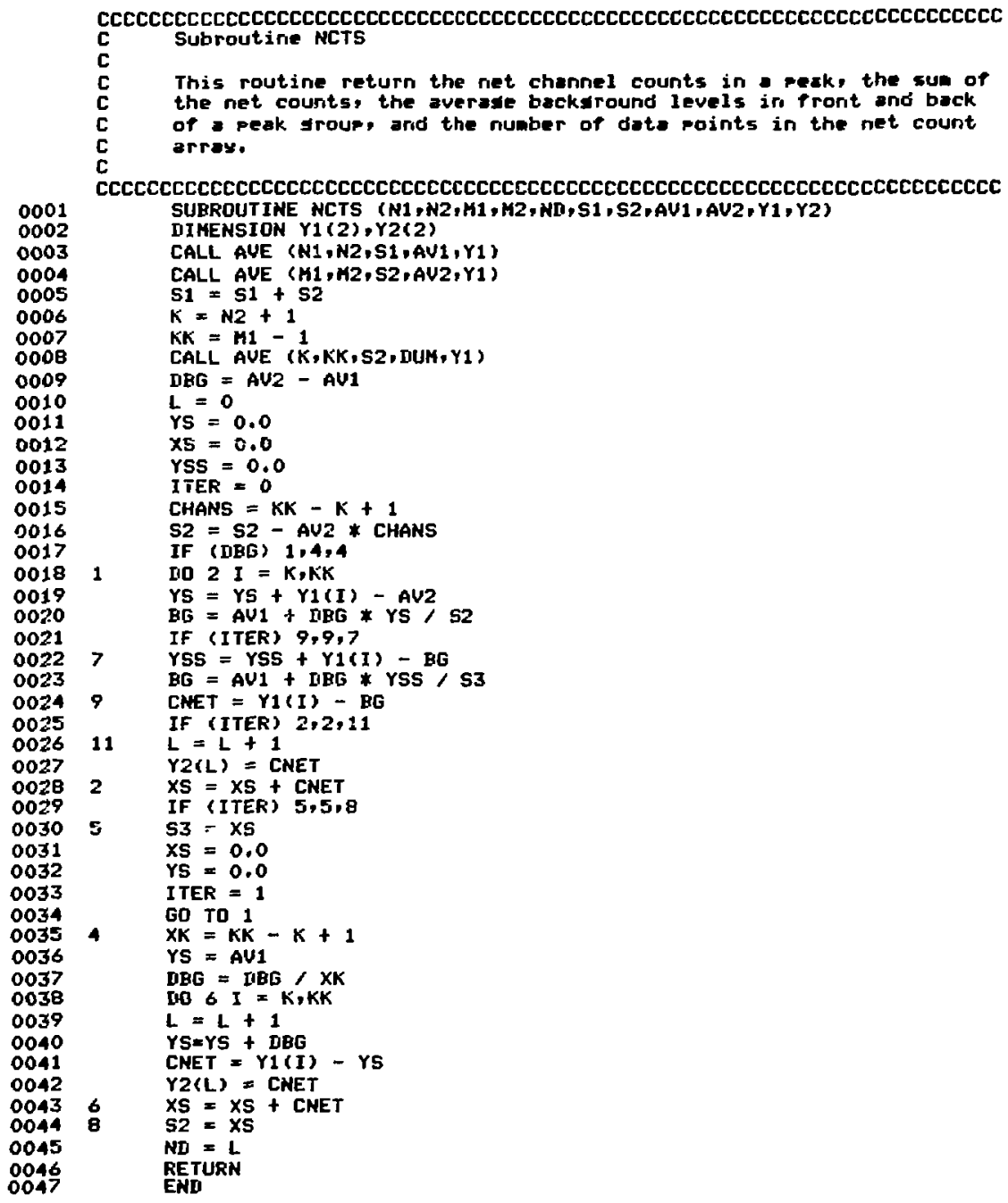




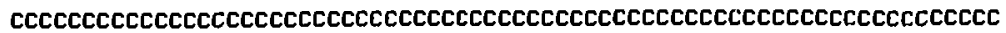

C

C SUBFOUTINE AVE

C THIS ROUTINE IETERHINES THE AUERAGE COUNT OF A GROUP OF IIATA

C VALUES FROH NS TO NE IMCLUSTUE. IT ALSO RETURNS THE SUH.

0001

0002

0003

0004

0005

0006

0007

0008

0009 SUBROUTINE AUE (NS, NE, SUH, AU,Y)

DIMENSION $Y(2)$

SUM $=0.0$

$S M=N E-N S+1$

DO $2 \mathrm{I}=\mathrm{NS}, \mathrm{NE}$

2 SUM $=$ SUH $+Y(I)$

AU $=$ SUM $/$ SH

RETURN

END 


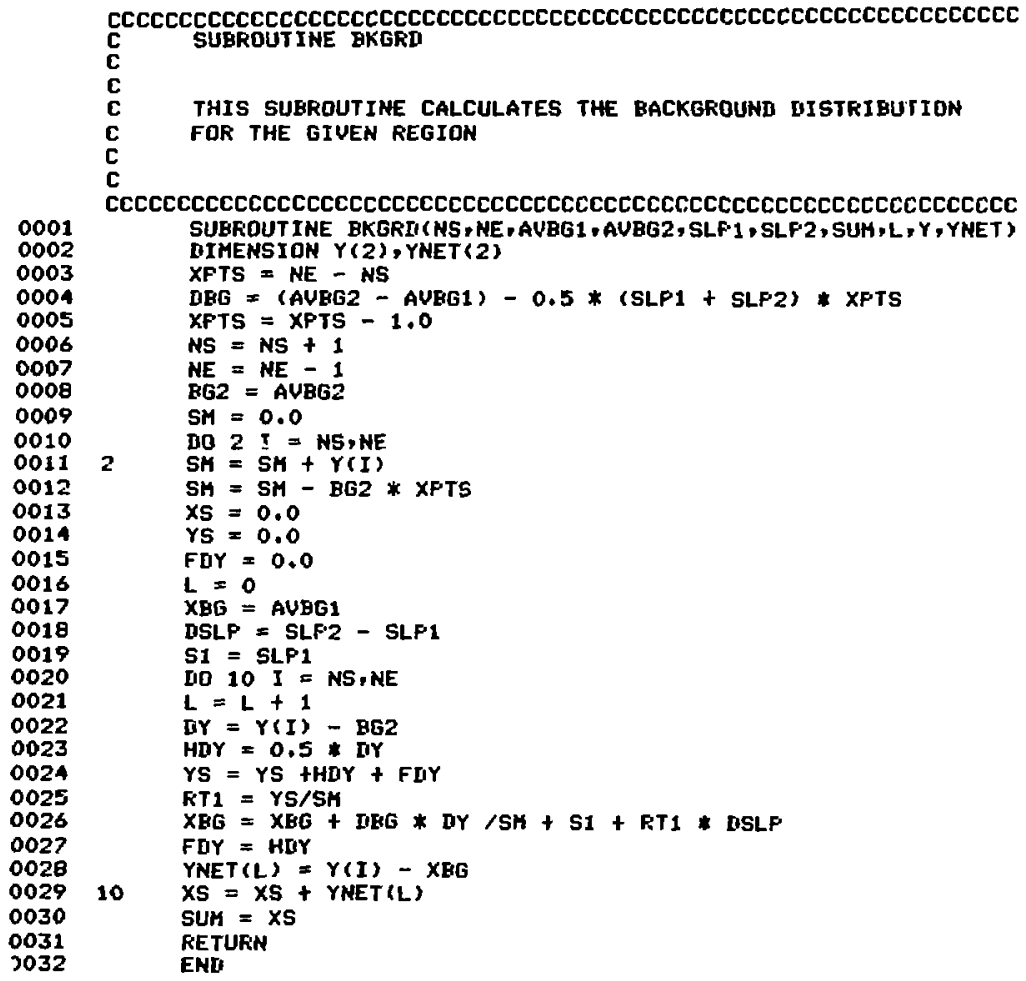




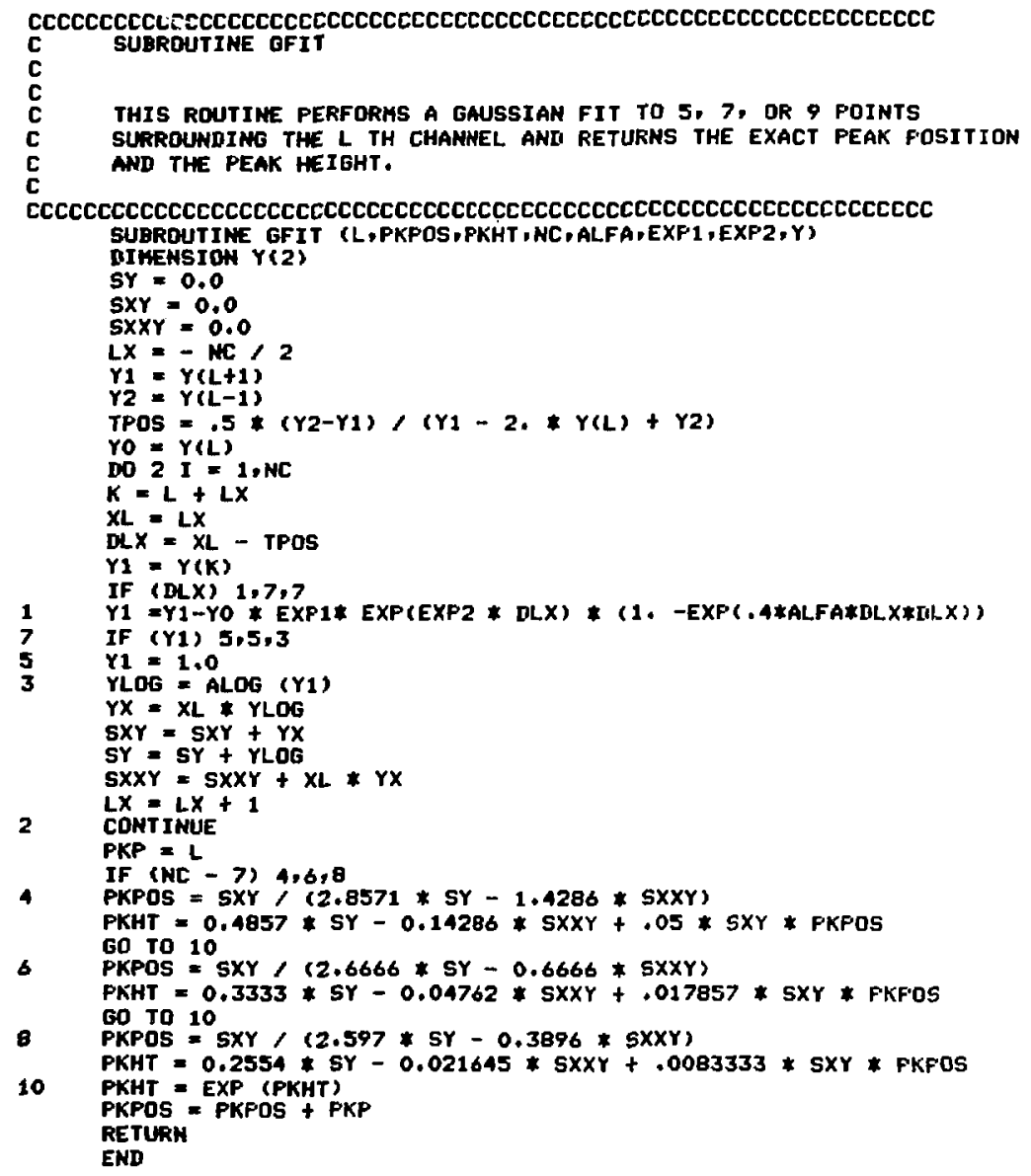




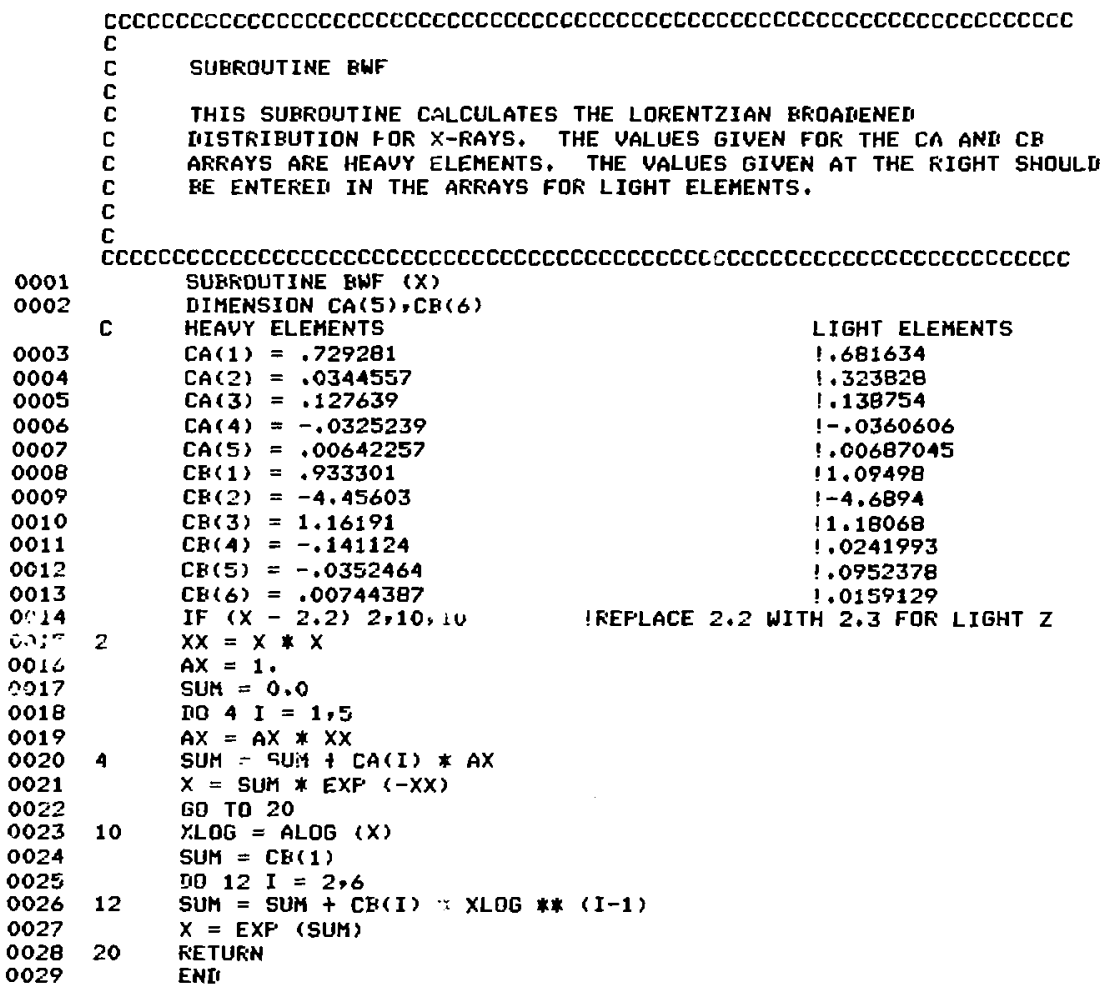




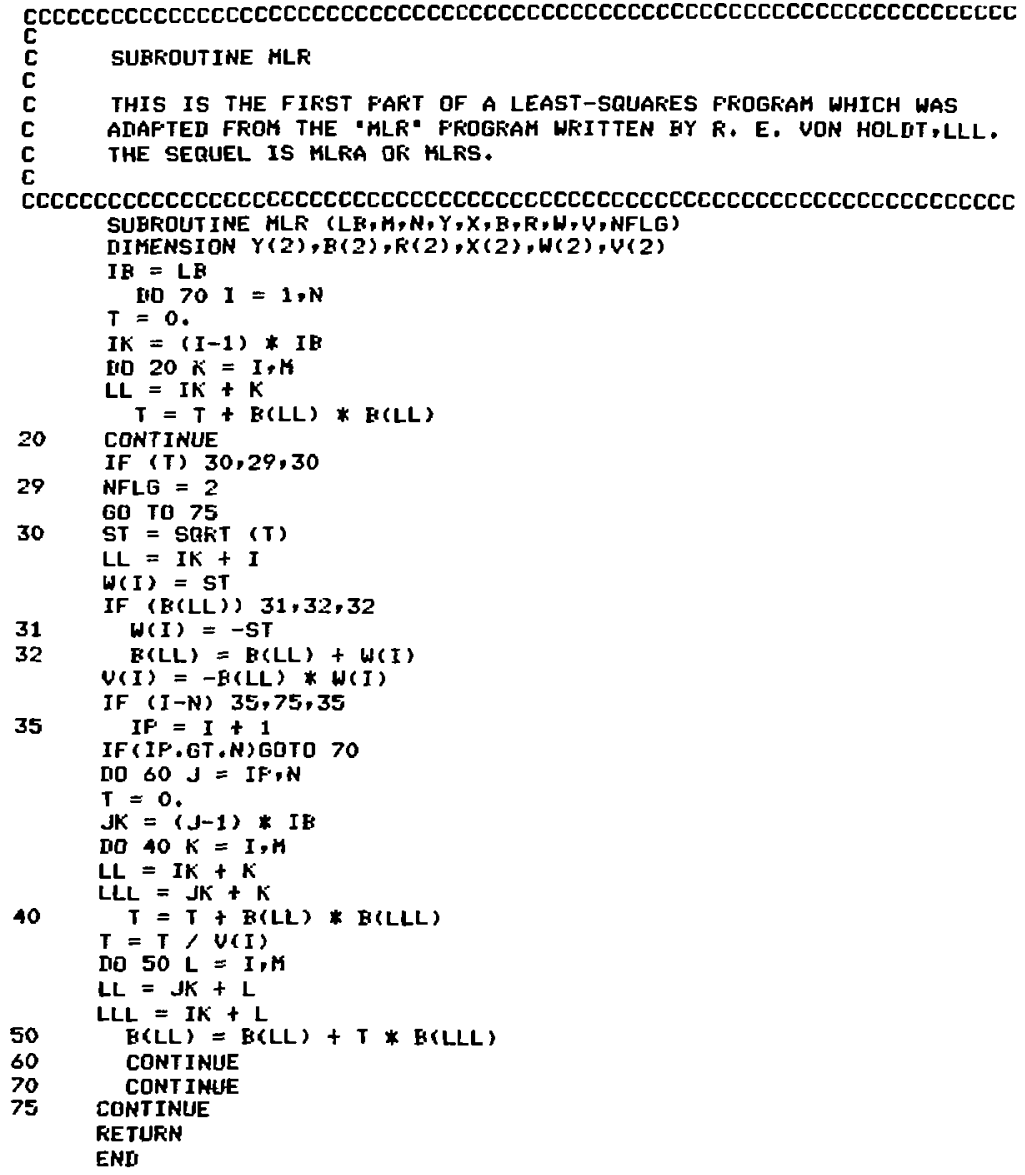




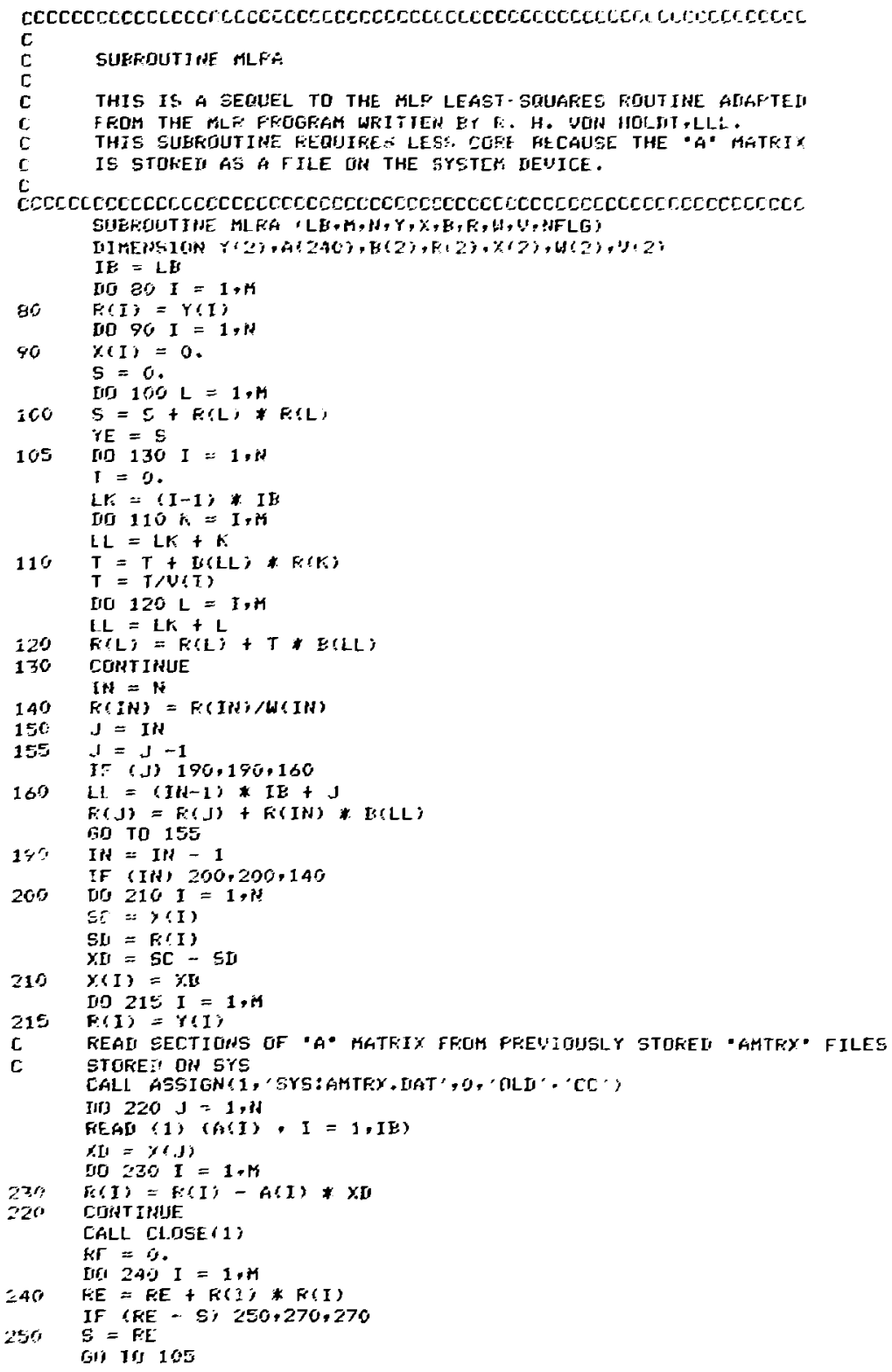




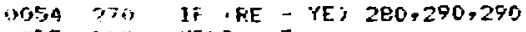

urist :'s PA REG $=3$

iRE "BS PETUFN

iU?'. ENI 


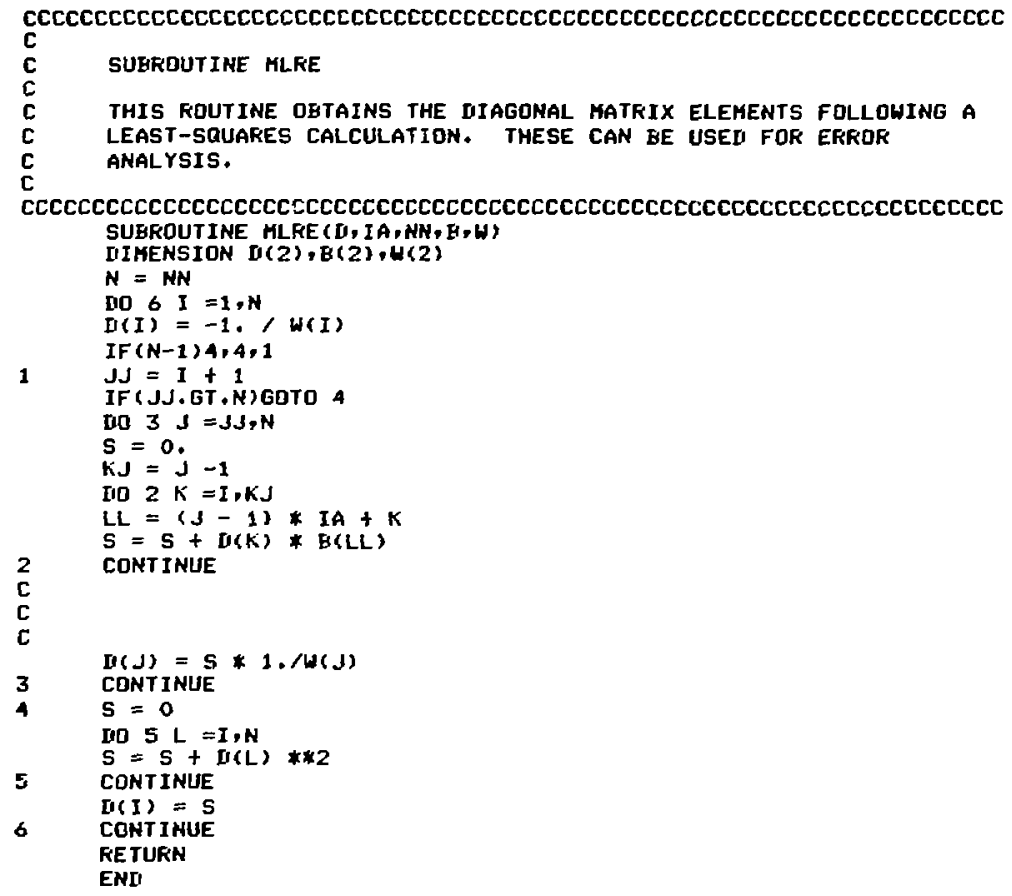




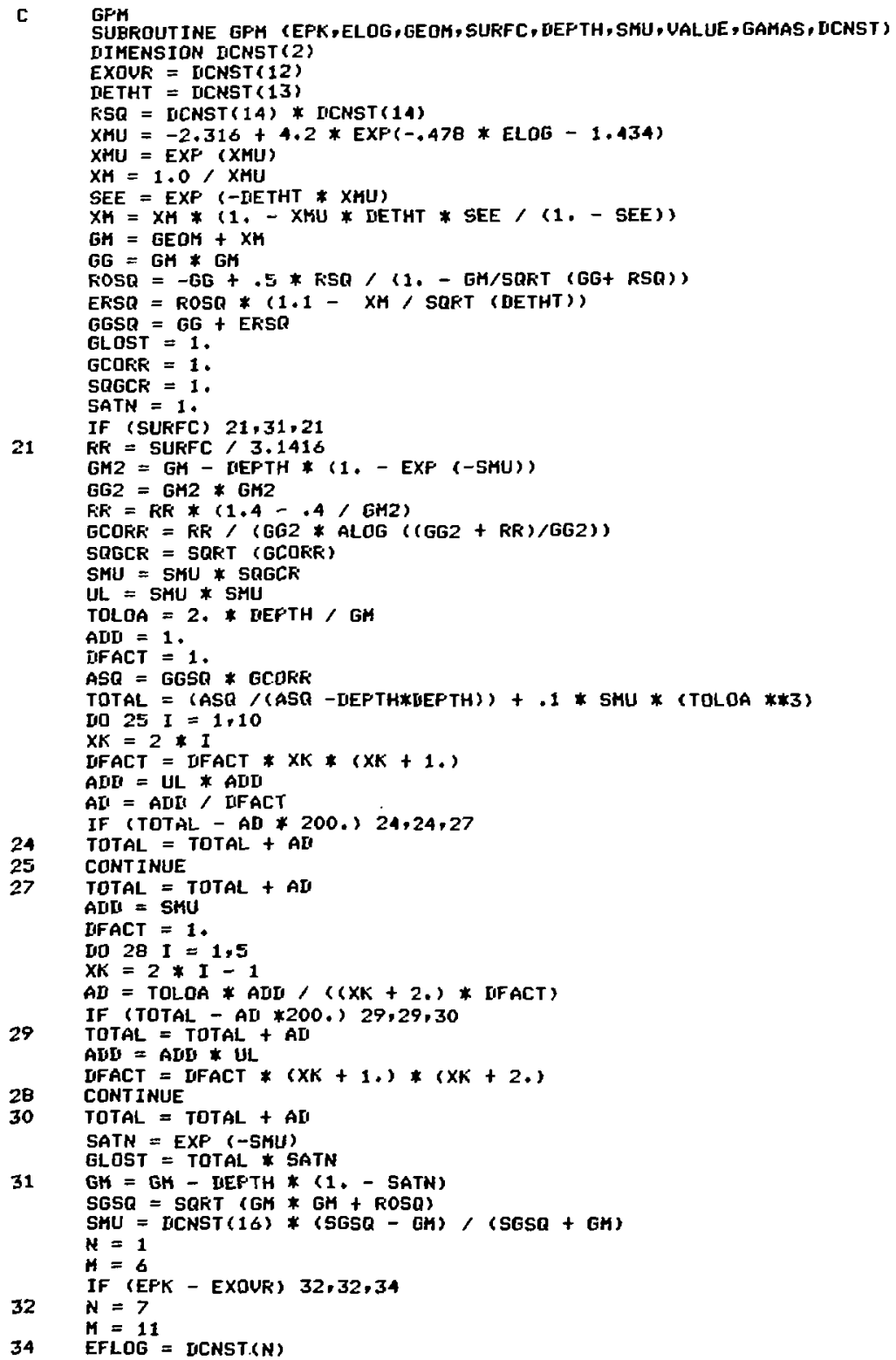


0065

0066

0067 0068 0069 0070 0071 0072

0073 $L=N+1$ DQ $36 I=\operatorname{LiH}$

36 EFLOG $=$ EFLOG + DCNST (I) * ELOG \$\# (I-N) EFF = EXP (EFLOG)

GAKAS = VALUE * GGSR * GCORR / (EFF * GLOST)

RD $=.7$ DCNST (14)

VALUE $=(1 .+R D /$ GG $) *$ SQGCR

RETURN

END 


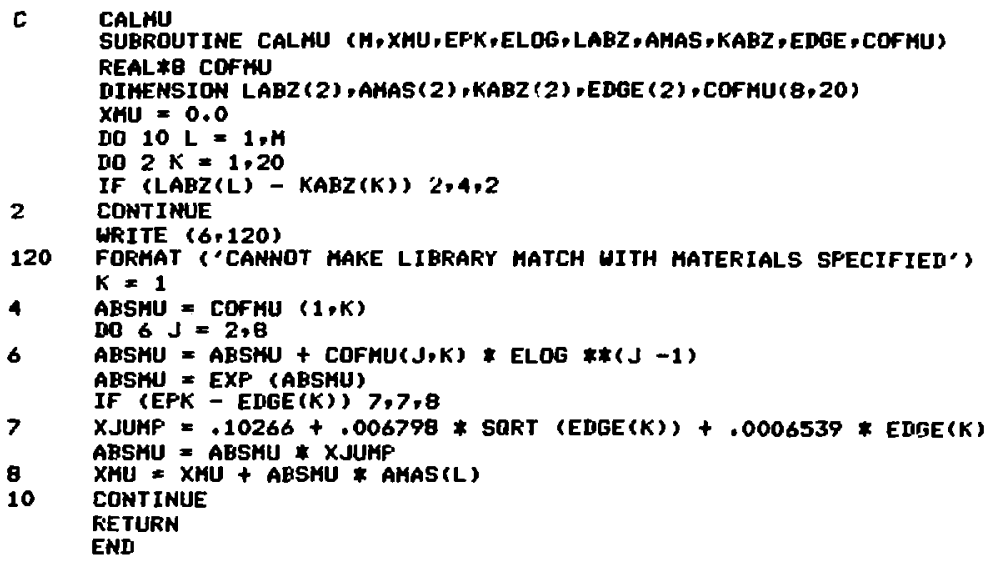


c

0001

0002

0003

0004

0005

0006

0007

0008

0009

0010

0011

0012

0013

0014

0015

0016

0017

0018

0019

0020

0021

0022

0023

CALGPM

SUEROUTINE CALGPH (EFK,VALUE)

REAL $* B$ COFHU

COMMON/PASS/ENJ ( 20 ), IST, IENE, NUMS J, NUME J, GAINJ,ZERO J, ST 1 J,

1 ST2J, STRJ (20), NENUM, ALF J, TAMF, TSLF, TLAKP, TLSLP, EKGSLF (2),

2 CFILE (3), SSS, YES, EK I P NGAIN, IKEGG , LISTF G, IIF ILE (3),

3 YSUM (20), QFT ( 100$)$, SUDF (20), SUMR (20), EIIGE (20), NSMBL (20),

4 COFHU $(8,20), M T L Z(5)$, CMPOS (5), NAES (5), AESRE (5), DCNST (16),

5 NF 1, NF 2, GEOM, SURFC, SHFWT, DEFTH

[IATA MTEL/'GE'/

ELOG $=$ ALOG $(.001 *$ EFK)

$5 M U=0.0$

IIEFTH $=0.5 *$ IIEPTH

IF (NF 1) 14,14,13

13 CALL CALHU (NF1,SHU,EFK,ELOG, MTLZ,CMPOS, NSHEL,ENGE, COFMU)

14 CALL GF'H (EFK, ELOG, GEOH, SURFC, IIEPTH,SMU,UALUE, GAMAS, IICNST)

IF (EFK - 500.) 9,9,10

C CORFECT FDR GE "IIEAI LAYEK" ATTENUATION.

9 CALL CALMU ( 1 , XMU,EFK,ELOG, MTKL, SHU, NSHEL, ENGE, COFMU)

GAMAS = GAMAS EXF (XHU)

10 IF (NF 2 ) $15.18,15$

$15 \quad$ ATN $=0.0$

CALL CALMU (NF 2, AKU, EF' , ELOG, NABS, AESRE, MSMBL, EIIGE, COFMU)

C ALJUST AESORREK ATTENUATIOH TO ACCOUNT FOR GAMMAS TRAUERSING

C THE ARSOREER AT SKEHEI ANGLES.

AHU = AMU * VALUE

IF (ANU - 6.9) 16,17,17

16 ATN = EXF (AMU)

17 GAMAS = GAMAS * ATN

18 UALUE = GAMAS

RETURN

ENI 